University of Nebraska - Lincoln

DigitalCommons@University of Nebraska - Lincoln

7-1-2007

\title{
Electronic, magnetic and transport properties of rare-earth monopnictides
}

\author{
Chun-Gang Duan \\ University of Nebraska-Lincoln, cgduan@clpm.ecnu.edu.cn \\ Ildar F. Sabiryanov \\ University of Nebraska-Lincoln, isabirianov2@unl.edu \\ Wai-Ning Mei \\ University of Nebraska-Lincoln, physmei@unomaha.edu \\ Peter A. Dowben \\ University of Nebraska-Lincoln, pdowben@unl.edu \\ Sitaram Jaswal \\ University of Nebraska, sjaswal1@unl.edu \\ See next page for additional authors
}

Follow this and additional works at: https://digitalcommons.unl.edu/physicsdowben

Part of the Physics Commons

Duan, Chun-Gang; Sabiryanov, Ildar F.; Mei, Wai-Ning; Dowben, Peter A.; Jaswal, Sitaram; and Tsymbal, Evgeny Y., "Electronic, magnetic and transport properties of rare-earth monopnictides" (2007). Peter Dowben Publications. 186.

https://digitalcommons.unl.edu/physicsdowben/186

This Article is brought to you for free and open access by the Research Papers in Physics and Astronomy at DigitalCommons@University of Nebraska - Lincoln. It has been accepted for inclusion in Peter Dowben Publications by an authorized administrator of DigitalCommons@University of Nebraska - Lincoln. 


\section{Authors}

Chun-Gang Duan, Ildar F. Sabiryanov, Wai-Ning Mei, Peter A. Dowben, Sitaram Jaswal, and Evgeny Y. Tsymbal 


\title{
Electronic, magnetic and transport properties of rare-earth monopnictides
}

\author{
Chun-Gang Duan ${ }^{1,2}$, R. F. Sabirianov ${ }^{2,3}$, W. N. Mei ${ }^{2,3}$, P. A. \\ Dowben $^{1,2}$, S. S. Jaswal ${ }^{1,2}$, and E. Y. Tsymbal ${ }^{1,2}$ \\ ${ }^{1}$ Department of Physics, University of Nebraska-Lincoln, Lincoln, NE 68588, \\ USA \\ ${ }^{2}$ Nebraska Center for Materials and Nanoscience, University of Nebraska-Lin- \\ coln, Lincoln, NE 68588, USA \\ ${ }^{3}$ Department of Physics, University of Nebraska at Omaha, Omaha, NE 68182, \\ USA \\ E-mail: wxbdcg@gmail.com
}

\begin{abstract}
The electronic structures and magnetic properties of many rare-earth monopnictides are reviewed in this article. Possible candidate materials for spintronics devices from the rare-earth monopnictide family, i.e. high polarization (nominally half-metallic) ferromagnets and antiferromagnets, are identified. We attempt to provide a unified picture of the electronic properties of these strongly correlated systems. The relative merits of several ab initio theoretical methods, useful in the study of the rare-earth monopnictides, are discussed. We present our current understanding of the possible half-metallicity, semiconductor-metal transitions, and magnetic orderings in the rare-earth monopnictides. Finally, we propose some potential strategies to improve the magnetic and electronic properties of these candidate materials for spintronics devices.
\end{abstract}

\section{Contents}

1. Introduction

2. Early experiments and theoretical studies 3

3. Recent theoretical and experimental progresses 5

3.1. Self interaction correction (SIC)

3.2. $\mathrm{LSDA}+U \quad 6$

3.3. GW approximation (GWA)

3.4. Dynamical mean-field theory (DMFT) 8

$\begin{array}{ll}\text { 3.5. Other theoretical approaches } & 9\end{array}$

3.6. Experimental studies $\quad 9$ 
4. Electronic, magnetic and transport properties 10

4.1. Gd monopnictides 12

4.2. Eu monopnictides 17

4.3. Er monopnictides 20

5. Half-metallicity and other interesting phenomena 20

5.1. Origins of half-metallicity 20

5.2. Metal-insulator transitions $\quad 22$

5.3. Magnetic ordering $\quad 22$

5.4. Spin-orbit coupling $\quad 25$

6. The future possibilities $\quad 26$

6.1. Possible half-metallic antiferromagnets 26

6.2. Enhancement of the transition temperatures and potential device applications 27

7. Conclusions

Acknowledgments 28

$\begin{array}{ll}\text { References } & 28\end{array}$

\section{Introduction}

Half-metallic ferromagnets, which were first so named by de Groot et al in 1983 [1], have attracted interest from both experimentalists and theorists $[2,3]$. Currently, most of the nominally half-metallic ferromagnets found are compounds that involve transition metal elements, for example $\mathrm{CrO}_{2}, \mathrm{Fe}_{3} \mathrm{O}_{4}, \mathrm{Co}_{2} \mathrm{MnSi}$ [3], and they suffer from a number of deficiencies that limit their application as high polarization materials in spintronics. The electronic structures of nominally half-metallic systems are, nonetheless of considerable interest as an avenue for studying the interplay between high polarization and band structure. Their attraction remains in spite of the growing recognition that true half-metallic character is unlikely to be ever demonstrated at finite temperatures, due to magnons $[4,5]$, as well as zero-temperature interactions $[6,7]$. It is natural, therefore, to explore the rareearth compounds, as rare-earth elements generally have much larger magnetic moments and demonstrate some fascinating phenomena $[8,9]$.

Rare-earth elements are chemically very similar due to an almost identical outer electron arrangement [10]. It remains, however, difficult to obtain impurity-free single crystals of the rare-earths or rare-earth compounds, and this may be responsible for some of the long-standing controversies concerning their electronic structure, transport properties and magnetic properties.

The rare-earths do, however, have different occupation numbers for the shallow inner $4 \mathrm{f}$ shell, ranging from 0 to 14 through the series $\mathrm{La}$ to $\mathrm{Lu}$. This changing $4 \mathrm{f}$ occupation means that the rare-earth elements and their compounds have a wide range of different magnetic properties and electronic structures. Due to the unfilled 4f shells of rare-earth atoms, it is a challenging problem to obtain an accurate theoretical description of the electronic structure of rare-earth compounds [11]. In spite of the fact that the $4 \mathrm{f}$ energy levels often overlap with the non- $4 \mathrm{f}$ broad bands of the system, they generally form very narrow resonances, and are often treated as core states in the theoretical efforts. Due to the highly localized nature of the $4 \mathrm{f}$ electrons, the direct $\mathrm{f}-\mathrm{f}$ interactions between neighboring rare-earth atoms are generally considered to be nearly negligible. However, there is evidence that this general belief has to be modified. For instance, in the cases of cerium (uranium) compounds [12-16], 4f (5f) level dispersion was observed experimentally, suggesting smaller f level localization in these systems. The unoccupied $f$ states certainly will adopt all the trappings of band structure in every conventional sense. 
f orbital moments generally cannot be quenched by the crystal field. Therefore the total magnetic moments have both orbital and spin components, and spin-orbital interactions are particularly strong for many of the rare-earth elements and compounds. These inner shell magnetic moments are largely aligned through intra-atomic $s(d)-f$ exchange interaction and weaker inter-atomic s-s (d-d) exchange interactions; thus the rare earth pnictide magnetic transition temperatures are generally much lower than those of $3 \mathrm{~d}$ transition metal elements or compounds. In spite of the increasingly compelling evidence for band structure [12], the $4 \mathrm{f}$ bands are generally very narrow, significantly different from the bands dominated by $\mathrm{s}, \mathrm{p}$ and $\mathrm{d}$ states. Therefore there exist strong on-site Coulomb repulsions between the highly localized f electrons $[17,18]$. This makes the independent particle approximation no longer valid and calculations based on local spin density approximation (LSDA) fail to describe the rare-earth $4 \mathrm{f}$ electrons correctly. To explain the behaviors of rare-earth $4 \mathrm{f}$ electrons, many-body effects must be taken into account and more accurate approximations or calculations beyond LSDA are absolutely necessary.

With the availability of better quality single crystals and thin films, together with the tremendous theoretical efforts in combining many-body theory and density functional theory (DFT) in the last 20 years, a better picture of electronic structure and magnetic properties of rare-earth elements and their compounds has taken shape. It is the major objective of this review to provide a detailed account of the progress made in the field of rare-earth monopnictides, $\mathrm{RX}(\mathrm{X}=\mathrm{N}, \mathrm{P}, \mathrm{As}, \mathrm{Sb}, \mathrm{Bi})$. This particular family is chosen because there are more than 50 members that crystallize in the simple NaCl-type structure, making the rare-earth monopnictides excellent candidates for both experimental and theoretical analysis.

In providing an overview of the electronic, magnetic and transport properties of rareearth monopnictides, we have given some preference to GdN and EuN. GdN and EuN are considered to be the most promising nominally half-metallic ferromagnets in the RX family. Attracting our attention are the mechanisms for magnetic ordering, the pressure/strain and impurity effects.

\section{Early experiments and theoretical studies}

Studies of the rare-earth elements and their compounds can be traced back more than 70 years. The agreement between experiment and calculations made by Van Vleck and Frank [19] on the effective magneton number of the rare-earth ions was regarded as one of the most successful applications of quantum mechanics to magnetism [20]. The surge in the study of rare-earth compounds in the 1960s was mainly motivated by a search for new ferromagnetic semiconductors [21-27]. Researchers began realizing that it was too complicated to interpret the experimental results if crystal distortions or impurities were considered. Thus, investigations on rare-earth monopnictides gradually became one of the most important directions in the study of rare-earth compounds, mostly because these binary compounds crystallize into the simple NaCl-type structure and were supposed to be more amenable to theoretical analyses [21].

Nevertheless, even with such a simple structure, the full understanding of rare-earth monopnictides' electronic structure and magnetic properties is still not complete. Rareearth monopnictides demonstrate rich magnetic orderings and their electronic structures are sensitive to external pressure and impurities. Due to the poor computational power and limited theoretical methods, earlier theoretical studies were restricted to analytical model calculations, such as crystalline field [23], effective-point-charge [28], d-f Coulomb interaction [29] and p-f mixing [30]. These calculations could explain some phenomena for certain rare-earth monopnictides, but the overall agreement with experiments for the whole $\mathrm{RX}$ family was not satisfactory. 
The earliest ab initio band structure calculation of the rare-earth monopnictides was carried out by Hasegawa and Yanase in 1977 [31]. They adopted an augmented planewave method with Slater Xa exchange potential [32] to calculate the energy bands of $\mathrm{GdX}(\mathrm{X}=$ $\mathrm{N}, \mathrm{P}, \mathrm{As}, \mathrm{Sb}$ ), in a self-consistent way. To deal with the $4 \mathrm{f}$ electrons, they used the frozencore approximation, i.e. the $4 \mathrm{f}$ electrons are treated as core electrons. On the basis of their calculations, Hasegawa and Yanase claimed that GdN is a semiconductor with an adjusted band gap of $1 \mathrm{eV}$, and other $\mathrm{Gd}$ monopnictides are semimetallic. But they also realized that if spin polarization is taken into account, then the $5 \mathrm{~d}-4 \mathrm{f}$ exchange splitting, which was estimated also to be around $1 \mathrm{eV}$, could make GdN exhibit metallic character as well [31].

In 1988, using a tight-binding model with parameters chosen to fit the band structures calculated by Hasegawa and Yanase [31], Narita studied the magnetic susceptibilities and spin structures of $\mathrm{GdX}$ [33]. This was the first attempt to understand the magnetic properties of rare-earth monopnictides from band structure calculations, though Narita obtained wrong magnetic ground states for these compounds. Using a similar method, Xia et al [34] studied the electronic structure of the GdAs/GaAs superlattice in 1991.

The first spin-polarized ab initio calculations on rare-earth monopnictides was provided by Petukhov et al in 1994 [35]. Using the linear-muffin-tin-orbital (LMTO) method within LSDA and treating the Er $4 \mathrm{f}$ states as localized corelike states with fixed spin occupancies, Petukhov and coworkers calculated the electronic structure of ErAs and $\mathrm{Er}_{x} \mathrm{Sc}_{1-x}$ As alloys. Later, in 1996, they studied extensively the electronic structures, equilibrium lattice constants, cohesive energies, bulk moduli and magnetic moments of GdX and $\operatorname{ErX}(X=N, P$, As) [36]. They found that the corelike treatment is satisfactory for most purposes not involving the $4 \mathrm{f}$ electrons directly. They also found that the electron band exchange splittings of the nitrides are significantly larger than those for the arsenides and phosphides, rendering GdN metallic for one spin channel and semiconducting for the other. Thus, Petukhov, Lambrecht, and Segall were actually the first to claim that GdN could be half-metallic, within their calculation scheme, which did not involve the $4 \mathrm{f}$ states explicitly. There have been other theoretical studies on Gd compounds; for example, Kasuya and Li tried f-d mixing and $\mathrm{f}-\mathrm{d}$ exchange interactions to explain the strong ferromagnetism in $\mathrm{GdN}$ in 1997 [37].

The early experimental reports on rare-earth monopnictides are widely scattered in the literature. For reviews on experimental studies before 1970 s, one can refer to $[25,38]$ and [39], but focusing on the experimental studies on the electronic, transport and magnetic properties, in the period from 1970s to 1990s, provides an insight into the parallel growth of materials science and electron spectroscopy. In the rare-earth monopnictide family, the Gd monopnictides have been extensively investigated. This is because Gd is located in the middle of the rare-earth group in the periodical table, and the $\mathrm{Gd} 4 \mathrm{f}$ orbitals are exactly half occupied; hence the orbital angular momentum is zero, as the ground state of $\mathrm{Gd}^{3+}$ is ${ }^{8} \mathrm{~S}_{7 / 2}$. Thus the spin-orbit, multipole, $\mathrm{p}-\mathrm{f}$ and $\mathrm{d}-\mathrm{f}$ mixing interactions are small [33], greatly simplifying the problems associated with electronic structure. In addition, the $\mathrm{Gd}$ monopnictides (and Gd metal) generally have much higher transition temperatures, which is also attractive in many respects [38].

Kaldis reported the first successful growth of large single crystals of GdP in 1974 [40]. This opened the door to a reliable determination of the electronic structure of GdP. Based on optical investigation [41], Güntherodt et al deduced that the crystal-field splitting of the $\mathrm{Gd} 5 \mathrm{~d}$ states is about $1.8 \mathrm{eV}$ and the positions of occupied Gd 4f levels in GdP are about $7 \mathrm{eV}$ below $E_{\mathrm{F}}$. They also found that GdP exhibits metal-like conductivity. Later experiments on GdN were controversial: GdN was reported as a semiconductor [42] and a semimetal [43]. Studies on the magnetic exchange interaction on Gd compounds clearly demonstrated that the free carriers contribute to a Ruderman-Kittel-Kasuya-Yosida (RKKY) [44-46] indirect exchange interaction [47, 48]. 
Urban et al [49] observed a large variation of the thermal broadening of the electron spin resonance (ESR) linewidth of Gd across the pnictides in 1978. They claimed that the conduction electrons exhibit almost $\mathrm{d}$ character; thus the exchange interaction depends on the amount of overlapping between $d$ wave functions. Palmstrøm et al [50] successfully grew single-crystal ErAs films on GaAs in 1988. Later magneto-transport measurements showed that ErAs is a low-density semimetal that magnetically orders at low temperature $(4.5-4.8 \mathrm{~K})$ [51].

In 1990, Degiorgi et al reported on results obtained from large single crystals of cubic and stoichiometric $\mathrm{YbN}$ [52]. They claimed that $\mathrm{YbN}$ is a self-compensated semimetal with the occupied f states about $6 \mathrm{eV}$ below $E_{\mathrm{F}}$ and the empty f states about $0.2 \mathrm{eV}$ above $E_{\mathrm{F}}$. Their later studies confirmed the semimetallic nature of $\mathrm{YbP}$ and $\mathrm{YbAs}$ [53]. Chattopadhyay et al carried out high-pressure magnetization and neutron-diffraction experiments on $\mathrm{CeSb}$ in 1994 [54]. They found that the magnetic ordering of CeSb is very sensitive to hydrostatic pressure. Waldfried et al used angle-resolved photoemission to study the electronic structure of dissociatively chemisorbed nitrogen on $\mathrm{Gd}(0001)$ in 1995 [55]. Xiao and Chien found that GdN films are insulating [56]. Li et al reported the growth of large single crystals of $\mathrm{GdX}(\mathrm{X}=\mathrm{P}, \mathrm{As}, \mathrm{Sb}, \mathrm{Bi})$ in 1996 and found them all to be well-compensated semimetals that order antiferromagnetically $[57,58]$. The photoemission studies of Yamada et al showed that the occupied $4 \mathrm{f}$ states in GdP lie around $8.4 \mathrm{eV}$ below $E_{\mathrm{F}}$, and shift down from GdP to GdBi [59]. The experiments on polycrystal GdN confirmed that stoichiometric $\mathrm{GdN}$ is ferromagnetic with a transition temperature $58 \mathrm{~K}$ [60].

\section{Recent theoretical and experimental progresses}

Recent theoretical progress in the rare-earth monopnictide studies follows the development of electronic structure calculations in solids. For strongly correlated systems, the accurate evaluations require going beyond the LSDA scheme. The following methods have been developed to overcome the deficiency of LSDA calculations.

\subsection{Self interaction correction (SIC)}

Despite its impressive successes [61], LSDA has an intrinsic deficiency, i.e. the self-interaction energy problem [62]. The self-Coulomb and self-exchange interactions in LSDA do not cancel completely, as in the case of Hartree-Fock approximation. This means that the LSDA will fail to correctly describe systems with strong electron-electron interactions. Numerous efforts have been made to address this problem [62-65]. In the self-interactioncorrected local-spin density approximation (SIC-LSDA) [65], the non-physical electron self-interactions, including both SIC Coulomb and corresponding SIC exchange-correlation terms, are subtracted from the LSDA Hamiltonian, and the energy functional is written as

$$
E_{\mathrm{SIC}}=E_{\mathrm{LSDA}}-\sum_{i}^{\mathrm{occ}}\left(\int \frac{n_{i}(\mathbf{r}) n_{i}\left(\mathbf{r}^{\prime}\right)}{\left|\mathbf{r}-\mathbf{r}^{\prime}\right|} \mathrm{d}^{3} r \mathrm{~d}^{3} r^{\prime}+\int n_{i}(\mathbf{r}) \varepsilon_{\mathrm{xc}}\left(n_{i}(\mathbf{r}), 0\right) \mathrm{d}^{3} r\right),
$$

where $E_{\mathrm{LSDA}}$ is the energy functional in the LSDA, $n_{i}(\mathbf{r})$ is the charge density corresponding to the $i$ th solution of the SIC-LSDA equation, and $\varepsilon_{\mathrm{xc}}\left(n_{\uparrow}, n_{\downarrow}\right)$ is the exchange-correlation energy density of a homogeneous system with spin densities $n_{\uparrow}$ and $n_{\downarrow}$. The SIC approach generates an orbital-dependent potential which can be significant for localized states, yielding a much-improved description of the static Coulomb correlation effect compared to that provided by the LSDA. The applications of SIC-LSDA are often quite successful [66-69].

Another advantage of the SIC-LSDA method is that the minimization of total energy, with respect to the number of localized electrons, leads to a determination of the nominal 
valence defined as the integer number of electrons available for band formation

$$
N_{\mathrm{v}}=\mathrm{Z}-N_{\text {core }}-N_{\text {SIC }}
$$

where $Z$ is the atomic number, and $N_{\text {core }}$ and $N_{\text {SIC }}$ are the number of core and localized (SIC) electron states, respectively. This information is important for the analyses of chemical properties of rare-earth compounds [67].

Application of the SIC-LSDA methodology to the rare-earth monopnictides was carried out by Aerts et al [70], Horne et al [71], and Szotek et al [72] in 2004. Together, these studies represent a systematic study of the electronic structure of 13 rare-earth nitrides, from $\mathrm{CeN}$ to $\mathrm{YbN}$. The calculations show that the rare-earths are trivalent in the mono-nitride ground state, with the exception of $\mathrm{Ce}$, which is tetravalent in $\mathrm{CeN}$. On the basis of the SIC-LSDA calculations, the claim is that these rare-earth nitrides display a wide range of electronic properties, despite having the same structure and similar lattice constants. Specifically, TbN, DyN and HoN are found to be narrow gap insulators, and CeN, ErN, $\mathrm{TmN}$ and $\mathrm{YbN}$ are metallic in both spin channels, while PrN, NdN, PmN, SmN, EuN and $\mathrm{GdN}$ are half-metallic ferromagnets in these ground-state calculations. The f-band manifold is split by the SIC into localized and band-like f electrons. Because of the different degree of hybridization between the rare-earth band-like $f$ and the nitrogen $p$ states, in the vicinity of the Fermi level, these compounds demonstrate different electronic properties. This is consistent with the electronic structures of $\operatorname{SmX}(X=N, P, A s, S b, B i)$ which have been studied by Svane et al [73]. Svane and co-workers found that the occupied $\mathrm{f}$ bands are formed in the vicinity of the Fermi level in all the SmX compounds.

\section{2. $\operatorname{LSDA}+U$}

In the LSDA, the potential is treated as an averaged orbital-independent one-electron potential. This may be a reasonable approximation for weakly correlated system, which corresponds to an extreme case of a Hubbard model [74] where the on-site Coulomb repulsion (Hubbard U) approaches zero. For strongly correlated systems like Mott insulators, however, ignoring the effective Coulomb parameter $U$ results in an incorrect prediction of the energy gap. The LSDA $+U[75-78]$ method was initially proposed to describe Mott insulators correctly. Following the Anderson model [79], electrons are separated into two subsystems: localized $d$ or $f$ electrons and delocalized $s$ or $p$ electrons. The $d-d(f-f)$ interaction is described by the Hubbard term $1 / 2 U_{\Sigma_{i \neq j}} n_{i} n_{j}$, where $n_{i}$ is the d(f)-orbital occupancy, instead of the LSDA averaged term (approximately) $E=U N(N-1) / 2$. Then a new energy functional can be written as

$$
E^{\mathrm{LSDA}+U}=E^{\mathrm{LSDA}}-\frac{1}{2} U N(N-1)+\frac{1}{2} U \sum_{i \neq j} n_{i} n_{j},
$$

and the orbital energies $\varepsilon_{i}$ are given by

$$
\varepsilon_{i}^{\mathrm{LSDA}+U}=\frac{\partial E^{\mathrm{LSDA}+U}}{\partial n_{i}}=\varepsilon_{i}^{\mathrm{LSDA}}+U\left(\frac{1}{2}-n_{i}\right) .
$$

This new formulation splits the orbital energies of occupied localized electrons $\left(n_{i}=1\right)$ and unoccupied localized electrons $\left(n_{i}=0\right)$ by $U$, thus reproducing the qualitatively correct physics for Mott-Hubbard insulators [77]. The above analysis is, however, only a simplified overview. The realization of the LSDA $+U$ scheme, in practice, requires much more theoretical effort [75] which is not addressed here. In passing, it is worth noting that a rotationally invariant form of the LSDA $+U$ functional is given by

$$
E^{\mathrm{LSDA}+U}=E^{\mathrm{LSDA}}+\frac{U-J}{2} \sum_{\sigma}\left[\operatorname{Tr} \rho^{\sigma}-\operatorname{Tr}\left(\rho^{\sigma} \rho^{\sigma}\right)\right],
$$


in which $\sigma$ is the spin, and $U$ and $J$ are the (spherically averaged) screened Coulomb electron- electron interaction (Hubbard parameter $U$ ) and Hund's rule exchange parameter (Stoner parameter $I$ ), respectively [80].

In practice, parameters $U$ and $J$ can be taken as adjustable parameters (for example, obtained through comparison with photoemission and inverse photoemission experiments). $U$ and $J$ can also be evaluated from the LSDA through the supercell LSDA approach [81]. Specifically, $U=F^{0}, J=\left(F^{2}+F^{4}\right) / 14$ for d electrons, and $J=\left(286 F^{2}+195 F^{4}\right.$ $\left.+250 F^{6}\right) / 6435$ for $\mathrm{f}$ electrons [78], where $F^{k}(k=0,2,4,6)$ are Slater integrals [82].

The first LSDA $+U$ calculation on the rare-earth monopnictides was carried out by Liechtenstein et al [83] in 1994. They studied the electronic structure and magneto-optical effects in CeSb. The Ce $4 \mathrm{f}$ level is found to be $2 \mathrm{eV}$ below the Fermi level and is hybridized with $\mathrm{Sb}$ p bands. Using the LMTO method, in 2003 Komesu et al [84] studied the electronic structure of ErAs and obtained band dispersions qualitatively similar with experiment. In 2004, Duan et al [85] considered the spin-orbit coupling in the LSDA $+U$ calculation of bulk ErAs, using the full-potential linear-augmented-plane-wave (FLAPW) method [86]. The theoretical $4 \mathrm{f}$ multiplet structure agrees well with photoemission experiments. In addition, clear evidence of $4 \mathrm{f}-5 \mathrm{~d}$ hybridization was found [86]. Later, with the same theoretical methodology, the electronic structure and magnetic ordering of $\mathrm{ErN}$ and ErAs was investigated [87].

In 2005, Duan et al reported on the effect of strain on the electronic and magnetic properties of GdN [88]. The results of LSDA $+U$ indicate that GdN is nominally a ground-state half-metal at the experimental lattice constant, but it may undergo a phase transition to a semiconductor with lattice expansion. Soon thereafter, these studies were extended to the electronic structure and magnetic ordering of the GdX monopnictides [89]. Duan et al provided a quantitative analysis of the RKKY and superexchange interaction [90] of the GdX monopnictide systems.

As previously indicated, a number of rare-earth pnictides appear to resemble groundstate half-metallic ferromagnets. Johannes and Pickett studied the electronic structure and magnetic exchange interactions in EuN and EuP also using the FLAPW method [91]. They found that EuN is a ground-state half-metallic ferromagnet within conventional LSDA $+U$ band theory. Ghosh et al studied the electronic, magnetic and optical properties of GdX in 2005 [92], with LMTO in the LSDA + U scheme. They also found that GdN is half-metallic if no further adjustment is implemented. Using LMTO within the LSDA + $U$ approach, Larson and Lambrecht [93] found that by putting the Hubbard $U$ on the d orbital as well, GdN is found to be a semiconductor; otherwise it is half-metallic, as noted by others $[70,88]$.

\subsection{GW approximation (GWA)}

Eigenvalues of the Kohn-Sham equations [94] are often interpreted as single-particle energies and are compared with photoemission spectra. This is not really justified and in many cases leads to incorrect predictions. A proper way to interpret the photoemission spectra is to use quasiparticle concepts [95]. The quasiparticle energies $E_{i}$ can be obtained [96] from

$$
\left[-\frac{1}{2} \nabla^{2}(\mathbf{r})+V(\mathbf{r})\right] \Psi_{i}(\mathbf{r})+\int \mathrm{d}^{3} r^{\prime} \Sigma\left(\mathbf{r}, \mathbf{r}^{\prime} ; E_{i}\right) \Psi_{i}\left(\mathbf{r}^{\prime}\right)=E_{i} \Psi_{i}(\mathbf{r}),
$$

where the self-energy $\Sigma$ addresses the effect of exchange and correlation and is intrinsically a non-local operator. The calculation of $\Sigma$ is, however, generally very difficult and requires some approximations. In the so-called $G W$ approximation (GWA) [97], $\Sigma$ is obtained by the Green's function method:

$$
\Sigma\left(\mathbf{r}, \mathbf{r}^{\prime}, E\right)=\frac{\mathrm{i}}{2 \pi} \int \mathrm{d} \omega G\left(\mathbf{r}, \mathbf{r}^{\prime} ; E-\omega\right) W\left(\mathbf{r}, \mathbf{r}^{\prime} ; \omega\right) ;
$$


here $G$ stands for the Green's function, and $W$ is the screened Coulomb interaction, which can be evaluated by the random phase approximation (RPA) [98], i.e. assuming the electrons are non-interacting when they respond to the external and induced field.

The application of GWA to realistic materials was first carried out by Hybertsen and Louie in the 1980s [99]. Godby et al [100] obtained similar results using same approach. Due to the complexity in calculating the self energy, the application of the GWA to rareearth compounds has not been undertaken until recently (for a recent review on GWA calculations, see [101]). Van Schilfgaarde et al [102] have drawn attention to their quasiparticle self-consistent GW (QSGW) calculations on Gd, GdP and GdAs. The QSGW calculations overestimate the position of the minority $\mathrm{Gd} \mathrm{f}$ shell by $\sim 4 \mathrm{eV}$. The details of these calculations are not yet available.

In 2000, using the LMTO method and assuming that the quasiparticle energy gap corrections scale inversely with the dielectric constant, Lambrecht [103] predicted that GdN is a narrow indirect band gap insulator. He also claimed that, by applying a magnetic field, the band gaps can be tuned by aligning the $\mathrm{Gd} 4 \mathrm{f}$ magnetic moments.

\subsection{Dynamical mean-field theory (DMFT)}

Dynamical mean-field theory (DMFT) is a more modern, non-perturbative method that has proven to be successful in investigating strongly correlated systems with local Coulomb interactions [104]. This theory was initially developed from the local impurity self-consistent approximation [105], which is the natural generalization of quantum many-body problems of the Weiss mean-field theory [106]. DMFT is believed to be a major step towards the reunion of two theoretical approaches, i.e. the DFT and many-body model Hamiltonian of condensed matter physics.

The spirit of DMFT is to map a lattice problem with many degrees of freedom onto an effective single-site (impurity) problem with fewer degrees of freedom. DMFT becomes exact in the limit of high lattice coordination numbers [105]. The underlying physical idea is that the dynamics, at a given site, may be considered to be the interaction of the degrees of freedom at this site with an external bath created by all other degrees of freedom on other sites, a dynamical mean-field approximation [106]. This impurity problem has to be solved self-consistently together with the $k$-integrated Dyson equation connecting the frequency-dependent self-energy $\Sigma(\omega)$ and the on-site Green function $G$ at frequency $\omega$ :

$$
G(\omega)=\frac{1}{V_{\mathrm{B}}} \int \mathrm{d}^{3} k \frac{1}{\omega+\mu-H_{\mathrm{LDA}}^{0}(\mathbf{k})-\Sigma(\omega)},
$$

where $\mu$ is the chemical potential, $H_{\mathrm{LDA}}^{0}$ is the one-particle Hamiltonian without the local Coulomb interaction, and $V_{\mathrm{B}}$ is the volume of the Brillouin zone.

Many techniques have been applied to solve the Anderson impurity model, such as quantum Monte Carlo, iterative perturbations, the fluctuation exchange approximation, the mean-field slave boson approach and the numerical renormalization group [104]. With these efforts, it is now possible to combine DMFT with modern electronic structure calculations to carry out LDA + DMFT [107] or spectral DFT [108] calculations.

The DMFT method has been successfully used to explain the $\alpha-\gamma$ transition in cerium, the $\delta$-phase of plutonium and several Mott insulators [104]. In 1998, Lægsgaard and Svane [109] calculated the excitation spectra of the Ce monopnictides CeN, CeP, and CeAs using the DMFT method, with parameters obtained from ab initio atomic and LMTO band structure calculations. The theoretical spectra are in good agreement with experiment. Recently, Sakai et al [110]also carried out a DMFT calculation on CeSb. 


\subsection{Other theoretical approaches}

There are also several calculations of rare-earth monopnictides using other approaches. Kalvoda et al studied the cohesive properties of GdN clusters using a quantum chemical (Hartree-Fock) method in 1998 [111]. They obtained a reasonable lattice constant for GdN (1.3-2.0\% larger than the experiment values), but the details concerning the electronic structure were not presented.

Combining a many-body model (multi-band Kondo lattice model) and 'ab initio' band structure calculations (LMTO), Santos et al studied the ferromagnetism and the temperature-dependent electronic structure of hexagonal close-packed (hcp) Gd in 2004 [112]. Later, using the same approach, Sharma and Nolting studied temperature-dependent electronic correlation effects in GdN in 2006 [113]. Assuming GdN to be a semiconductor, they obtained its quasiparticle spectral densities and density of states and found that the correlation effects were strongly temperature dependent. Using the $\mathrm{s}-\mathrm{f}$ model, Bhattacharjee and Jaya, in 2006 [114], studied correlation and temperature effects on the electronic structure of bulk and thin film GdN. Bhattacharjee and Jaya also found a red shift of the GdN conduction bands with respect to temperature. Without any special treatment of the 4f states, Landrum calculated the electronic structure of $\mathrm{CeN}$ in 1999 using the LMTO method [115].

In summarizing the various theoretical approaches, discussed above, all have their advantages and drawbacks. For instance, because of the localized nature of the strongly correlated electrons, most of the theoretical methods, except for the GWA, were first developed using the LMTO method, which is based on a local orbital basis, as a starting point. For $4 \mathrm{f}$ systems, the SIC + LSDA method sometimes overestimates the separation between the occupied and unoccupied $f$ states [70]. The LSDA $+U$ method now has been implemented into several band structure schemes, due to its relatively simple physics, and thus is more widely accepted. The Hartree-Fock approximation treatment in the LSDA+ $U$ scheme to the on-site Coulomb interactions is, however, too crude for strongly correlated systems [107]. This approach is successful in describing the long-range ordered insulating states of correlated systems, while it fails to describe correctly the strongly correlated paramagnetic states. GWA and DMFT methods are believed to be more accurate than many other strategies in describing the strongly correlated systems. At this stage, the GWA and DMFT approaches are still too cumbersome to be used by the whole theory community.

Nevertheless, concepts like the Hubbard $U$ and the self-energy $\Sigma$ are now being widely adopted [73, 107]. In addition, the Wannier functions make it possible to set up localized orbitals through a plane wave basis $[116,117]$. We expect that there will be many more $a b$ initio studies on strongly correlated systems over the next few years.

\subsection{Experimental studies}

A series of ESR measurements has been carried out on single crystals of GdAs [118], GdP [119] and GdBi [120] since 2000. The magnetic structure of these compounds was confirmed to be antiferromagnetic (AFM) type II. The Fermi surface and magnetic properties of TbSb were investigated by Nakanishi et al in 2004 [121] using de Hass-van Alphen and high-field magnetization measurements.

In 2005 Leuenberger et al [122] reported electronic and magnetic properties of highquality thin films of GdN. They found that a $500 \AA$ thick GdN film exhibits physical and magnetic properties close to that of bulk GdN. The element specific x-ray magnetic circular dichroism (XMCD) measurements clearly demonstrated that the $\mathrm{N} \mathrm{p}$ states are magnetically polarized. This agrees with theoretical predictions, as will be shown later. 


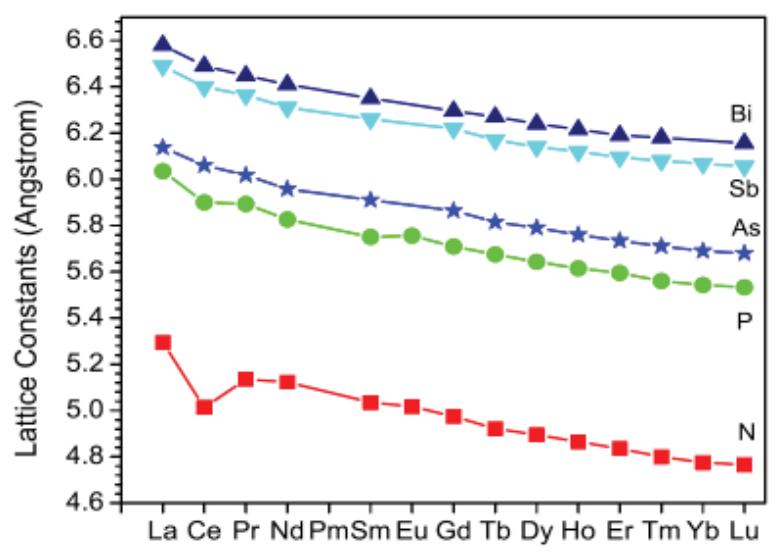

Figure 1. Lattice constants of rare-earth monopnictides: RN (squares), RP (circles), RAs (stars), RSb (down triangles), RBi (up triangles).

Leuenberger and co-workers also found that the electrical conductivity of GdN is thermally activated down to the ferromagnetic $T_{\mathrm{c}}$, below which GdN exhibits metallic character. They attribute the origin of this semiconductor-metal transition to a non-stoichiometric $\mathrm{GdN}$ film, similar to that found for $\mathrm{EuO}[123,124]$. Later experiments demonstrated a significant reduction of $T_{\mathrm{c}}$ as a result of the influence of lattice expansion [125], as predicted by theory [88]. More recently, Granville et al [126] claimed that GdN is semiconducting in both paramagnetic and ferromagnetic states $\left(T_{\mathrm{c}}=68 \mathrm{~K}\right)$. The suggestion was that the $\mathrm{N}$ vacancies may be responsible for the conductivity in GdN.

As a final note, although tangential to GdN, Leuenberger and co-workers [127] also found that $\mathrm{Fe}$ layers induce long-range magnetic order in $\mathrm{GdN}$ layer at temperatures above the $T_{\mathrm{c}}$ of $\mathrm{GdN}$ in $\mathrm{GdN} / \mathrm{Fe}$ multilayers.

\section{Electronic, magnetic and transport properties}

There are many similarities among the rare-earth monopnictide family, and some stunning differences with regard to metallicity and magnetic ordering. This is summarized in table 1, which lists the experimental lattice constants of all the rare-earth monopnictides, together with their magnetic ordering, transition temperatures and metallicity, i.e. insulating (semiconducting) or (semi)metallic, for most of the more than 60 compounds in the rareearth monopnictide family. Most of the rare-earth monopnictide family have the $\mathrm{NaCl}$ type structure (space group $F m \overline{3} m$ ). EuAs, EuSb and EuBi do not adopt the NaCl-type structure, and have been omitted in table 1. There are no experimental reports for the Pm monopnictides, most probably due to the fact that Pm must be artificially prepared. We are also unable to find any report on $\mathrm{YbBi}$.

From table 1, we can see that the lattice constants of rare-earth monopnictides, as a general trend, increase from $\mathrm{N}$ to $\mathrm{Bi}$ but decrease from $\mathrm{La}$ to $\mathrm{Lu}$ : the lattice constant typically decreases with increasing $4 \mathrm{f}$ occupancy, as indicated in figure 1 . This trend in the lattice constant can be simply explained by the increase of anion sizes and the decrease of cation sizes with the increase of atomic number. Not surprisingly, the smallest lattice constant belongs to $\mathrm{LuN}(4.766 \AA)$, and the largest to $\mathrm{LaBi}(6.580 \AA)$ : a difference of about $38 \%$. We note that there are exceptions to this general trend: for example, CeN, SmP. As shown in the experiments of Olcese [128] and theoretical calculations by Aerts et al [70], the jump in lattice constants from $\mathrm{CeN}$ to $\mathrm{PrN}$ is due to the fact that the ground state for $\mathrm{Ce}$ in $\mathrm{CeN}$ is tetravalent instead of trivalent. 
Table 1. Physical and magnetic properties of rare-earth monopnictides. Unless explicitly indicated, the data are collected from [39]. 'Metallicity' values are band gaps in eV derived from optical absorption of thin films if the material is an insulator or semiconductor. SC means semiconductor, SM means semimetal.

\begin{tabular}{|c|c|c|c|c|c|c|}
\hline & & $\mathrm{N}$ & $\mathrm{P}$ & As & $\mathrm{Sb}$ & $\mathrm{Bi}$ \\
\hline \multirow[t]{2}{*}{$\mathrm{La}$} & $a(\AA)$ & $5.295^{\mathrm{a}}$ & $6.035^{\mathrm{b}}$ & $6.137^{\mathrm{c}}$ & $6.490^{\mathrm{d}}$ & $6.577^{\mathrm{e}}$ \\
\hline & Metallicity & $\mathrm{SC}, 0.82$ & & & SC, $0.7-0.8$ & \\
\hline \multirow[t]{4}{*}{$\mathrm{Ce}$} & $a(\AA)$ & $5.013^{\mathrm{a}}$ & 5.90 & 6.06 & 6.40 & 6.49 \\
\hline & Ordering & $\begin{array}{l}\text { No order } \\
\text { down to } 1.5 \mathrm{~K}\end{array}$ & AFM-I & AFM-I & $\begin{array}{l}\text { AFM } \\
\text { (complicated) }\end{array}$ & $\begin{array}{l}\text { AFM } \\
\text { (complicated) }\end{array}$ \\
\hline & $T_{\mathrm{N}(\mathrm{C})}(\mathrm{K})$ & & $6-7,8.5,9$ & $5-7.5,7.5$ & 16,18 & $25,25.5,26$ \\
\hline & Metallicity & $\mathrm{SM}^{\mathrm{f}}$ & SC, 1.1 & SC, 0.7 & $\mathrm{SM}^{\mathrm{f}}$ & \\
\hline \multirow[t]{4}{*}{$\operatorname{Pr}$} & $a(\AA)$ & $5.135^{\mathrm{g}}$ & 5.893 & 6.018 & 6.364 & 6.448 \\
\hline & Ordering & & & & & \\
\hline & $T_{\mathrm{N}(\mathrm{C})}(\mathrm{K})$ & & & & & \\
\hline & Metallicity & SC, 1.03 & & SC, 1.0 & SC, 0.66 & \\
\hline \multirow[t]{4}{*}{$\mathrm{Nd}$} & $a(\AA)$ & $5.123^{\mathrm{a}}$ & 5.826 & 5.958 & 6.31 & 6.41 \\
\hline & Ordering & FM & AFM-I & AFM-I & AFM-I & AFM-I \\
\hline & $T_{\mathrm{N}(\mathrm{C})}(\mathrm{K})$ & $27.6,29,32,35$ & 7,11 & $10.6,12.5,13$ & $15.5,16$ & 24,25 \\
\hline & Metallicity & SC, 0.8 & & SC, 1.04 & & \\
\hline \multirow[t]{4}{*}{$\mathrm{Sm}$} & $a(\AA)$ & 5.035 & 5.75 & 5.91 & 6.26 & 6.35 \\
\hline & Ordering & AFM & AFM & AFM & AFM & AFM \\
\hline & $T_{\mathrm{N}(\mathrm{C})}(\mathrm{K})$ & $<2,13,18$ & 1.65 & 1.8 & $2.1,2.8$ & 8.6 \\
\hline & Metallicity & SC, 0.7 & $\mathrm{SC}, 1.09$ & $\mathrm{SC}, 1.03$ & SC, 0.59 & \\
\hline \multirow[t]{4}{*}{$\mathrm{Eu}$} & $a(\AA)$ & $5.017^{\mathrm{h}}$ & $5.756^{\mathrm{i}}$ & - & - & - \\
\hline & Ordering & & & & & \\
\hline & $T_{\mathrm{N}(\mathrm{C})}(\mathrm{K})$ & & & & & \\
\hline & Metallicity & SC, 0.76 & & & & \\
\hline \multirow[t]{4}{*}{ Gd } & $a(\AA)$ & $4.974^{i}$ & $5.709^{\mathrm{j}}$ & $5.864^{i}$ & $6.219^{j}$ & $6.295^{j}$ \\
\hline & Ordering & $\mathrm{FM}^{\mathrm{j}}$ & AFM-II & AFM-II' & AFM-II & AFM-II \\
\hline & $T_{\mathrm{N}(\mathrm{C})}(\mathrm{K})$ & $58.0^{\mathrm{i}}$ & $15.9^{\mathrm{j}}$ & $18.7^{\mathrm{j}}$ & $23.4^{i}$ & $25.8^{j}$ \\
\hline & Metallicity & SC, $0.98,0.85$ & $\mathrm{SM}^{\mathrm{j}}$ & $\mathrm{SM}^{\mathrm{j}}$ & $S M^{j}$ & $S M^{j}$ \\
\hline \multirow[t]{4}{*}{$\mathrm{Tb}$} & $a(\AA)$ & 4.922 & 5.675 & 5.815 & 6.17 & 6.27 \\
\hline & Ordering & $\mathrm{FM}, \mathrm{AFM}^{\mathrm{k}}$ & AFM-II & AFM-II & AFM-II & AFM-II \\
\hline & $T_{\mathrm{N}(\mathrm{C})}(\mathrm{K})$ & $T_{\mathrm{C}}: 34,39,42 T_{\mathrm{N}}: 31^{\mathrm{k}}$ & 8,9 & $10.5,12$ & $14,15,16.5$ & 17,18 \\
\hline & Metallicity & SC, 0.8 & & SC, 0.7 & $\mathrm{SC}, 0.35$ & \\
\hline \multirow[t]{4}{*}{ Dy } & $a(\AA)$ & 4.895 & 5.643 & 5.79 & 6.14 & 6.24 \\
\hline & Ordering & $\mathrm{FM}, \mathrm{AFM}$ & Flop-side FM & Flop-side FM & AFM-II & AFM-II \\
\hline & $T_{\mathrm{N}(\mathrm{C})}(\mathrm{K})$ & $T_{\mathrm{C}}: 17,17.6,26 T_{\mathrm{N}}: 15$ & $4.5,8$ & 8.5 & $9.5,12$ & 12,13 \\
\hline & Metallicity & SC, $0.91,0.95$ & SC, 25 & SC, $0.9,1.0$ & & \\
\hline \multirow[t]{4}{*}{ Ho } & $a(\AA)$ & 4.865 & 5.615 & 5.76 & 6.12 & 6.215 \\
\hline & Ordering & $\mathrm{FM}$ & Flop-side FM & AFM-II & AFM-II & AFM \\
\hline & $T_{\mathrm{N}(\mathrm{C})}(\mathrm{K})$ & $11,13.3,18$ & 5.5 & 4.8 & 5,9 & 6.7 \\
\hline & Metallicity & $\mathrm{SC}, 0.73,1.05$ & SC, $1.1-1.2$ & SC, 0.8 & SC, 0.45 & \\
\hline \multirow[t]{4}{*}{ Er } & $a(\AA)$ & $4.835^{\mathrm{a}}$ & 5.595 & 5.734 & 6.095 & 6.19 \\
\hline & Ordering & $\mathrm{FM}$ & AFM-II & AFM-II & AFM-II & AFM \\
\hline & $T_{\mathrm{N}(\mathrm{C})}(\mathrm{K})$ & $3.4,4.5,5,6$ & $2.3,3.1$ & $2.9,3.5$ & $3.55,3.7$ & $3.5,3.9$ \\
\hline & Metallicity & $\mathrm{SC}, 1.2,1.3$ & $\mathrm{SM}^{1}$ & & & \\
\hline \multirow[t]{4}{*}{$\mathrm{Tm}$} & $a(\AA)$ & 4.80 & 5.56 & 5.71 & 6.08 & 6.18 \\
\hline & Ordering & No order down to $1.3 \mathrm{~K}$ & & & & \\
\hline & $T_{\mathrm{N}(\mathrm{C})}(\mathrm{K})$ & & & & & \\
\hline & Metallicity & SC, 1.1 & & $\mathrm{SC}, 1.18$ & & \\
\hline
\end{tabular}


Table 1, continued.

\begin{tabular}{cllllll}
\hline & & $\mathrm{N}$ & $\mathrm{P}$ & $\mathrm{As}$ & $\mathrm{Sb}$ & $\mathrm{Bi}$ \\
\hline $\mathrm{Yb}$ & $a(\AA)$ & 4.775 & 5.543 & 5.69 & 6.067 & - \\
& Ordering & $\mathrm{AFM}$ & $\mathrm{AFM}$ & $\mathrm{AFM}$ & & \\
& $T_{\mathrm{N}(\mathrm{C})}(\mathrm{K})$ & $0.75^{\mathrm{m}}$ & $0.66^{\mathrm{m}}$ & $0.55^{\mathrm{m}}$ & & \\
& Metallicity & $\mathrm{SM}^{\mathrm{n}}$ & $\mathrm{SM}^{\mathrm{n}}$ & $\mathrm{SM}^{\mathrm{n}}$ & & \\
& $a(\AA)$ & $4.766^{\circ}$ & $5.533^{\mathrm{P}}$ & $5.68^{\mathrm{p}}$ & $6.056^{\mathrm{P}}$ & $6.156^{\mathrm{p}}$ \\
& Metallicity & $\mathrm{SC}, 1.55,1.6$ & & & & \\
\hline
\end{tabular}

${ }^{\mathrm{a}}$ Reference [128]. ${ }^{\mathrm{b}}$ Reference [130]. ${ }^{\mathrm{c}}$ Reference [131]. ${ }^{\mathrm{d}}$ Reference [132]. ${ }^{\mathrm{e}}$ Reference [133]. ${ }^{\mathrm{f}}$ Reference [134]. ${ }^{\mathrm{g}}$ Reference [135]. ${ }^{\mathrm{h}}$ Reference [136]. ${ }^{\mathrm{i}}$ Reference [137]. ${ }^{\mathrm{j}}$ Reference [60]. ${ }^{\mathrm{k}}$ Reference [138]. ${ }^{\mathrm{l}}$ Reference [51]. ${ }^{\mathrm{m}}$ Reference [139]. ${ }^{\mathrm{n}}$ Reference [53]. ${ }^{\circ}$ Reference [140]. ${ }^{\mathrm{p}}$ Reference [141].

The bonding between the rare-earth atoms and pnictogen atoms cannot be simply described as ionic or covalent, otherwise all the rare-earth monopnictide family should be insulators (semiconductors). However, from $\mathrm{N}$ to $\mathrm{Bi}$, we can see a gradual increase in metallicity in these compounds. The typical carrier concentrations in these compounds are around $10^{19}-10^{21} \mathrm{~cm}^{-3}$ [103], making them semimetals or highly doped n-type semiconductors.

In listing the known energy gaps for those rare-earth monopnictide compounds that are regarded as semiconductors, we note that the light pnictides, like the nitrides, tend to be larger energy gap semiconductors than the heavy pnictogen compounds. The changes in the energy gaps from La to Lu are rather scattered and it is hard to find a clear trend. We should point out that those data for energy gaps are not that reliable, since the electronic and transport properties are strongly affected by impurity dopants; thus a number of controversies exist in the comparison of various experiments. For example, GdP, GdAs and GdSb were thought to be semiconductors [39], but later experiments on high-quality samples clearly showed that they are actually semimetals [47, 57].

The magnetic ordering of rare-earth monopnictides is also quite complicated, ranging from ferromagnetic (FM), flip-flop FM [22], to AFM type II. Three typical AFM configurations (also see [129]) are depicted in figure 2. As we can see from table 1, most monopnictides have AFM ground states with exceptions among the nitrides and phosphides. Overall, we can see that the magnetic interactions gradually increase from La to $\mathrm{Gd}(\mathrm{FM}, 58 \mathrm{~K})$, then decrease again from $\mathrm{Gd}$ to $\mathrm{Lu}$. With the increase of anion sizes, the FM pnictides become AFM and the Néel temperature increases. Apparently, HoP and DyP are in the intermediate region between FM and AFM, as flip-flop FM can be regarded as combination of a FM and an AFM configuration [22]. This balance between $\mathrm{AFM}$ and FM ordering may also be the reason that $\mathrm{CeN}$ and $\mathrm{TmN}$ have no apparent net magnetic order.

The electronic structure calculations on three typical rare-earth pnictides, based on the LSDA $+U$ scheme with FLAPW method [86], confirm some of these trends and also can illustrate the effect of changing the lattice constant, without changing the pnictide, which is not easily done in experiment. For convenience, the Brillouin zone of the face-centered cubic (fcc) lattice is shown in figure 3, together with definitions of some high-symmetry $k$ points and $k$-lines.

\subsection{Gd monopnictides}

Among all the rare-earth monopnictides, the Gd monopnictides have been and continue to be studied extensively, nonetheless, there still remains intense controversy regarding their electronic structure and transport properties $[31,36,43,58,70,88,93,103,122,126]$. 


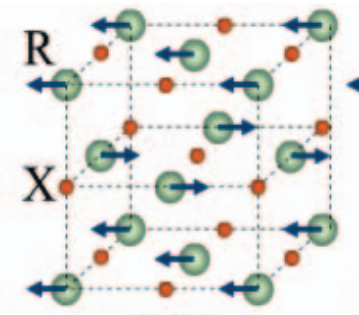

(a)

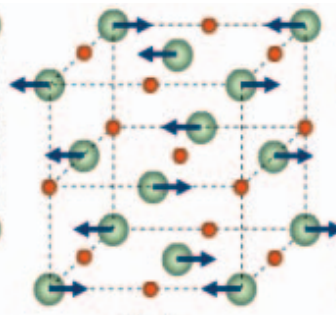

(b)

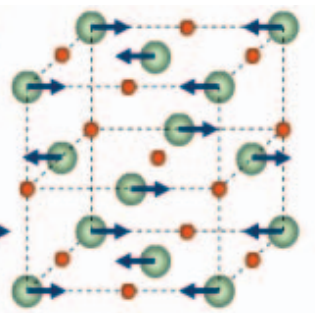

(c)

Figure 2. Three antiferromagnetic ordering configurations in rare-earth monopnictides: (a) AFM-I, (b) AFM-II, (c) AFM-III. Arrows indicate magnetic moment orientations on rareearth atoms.

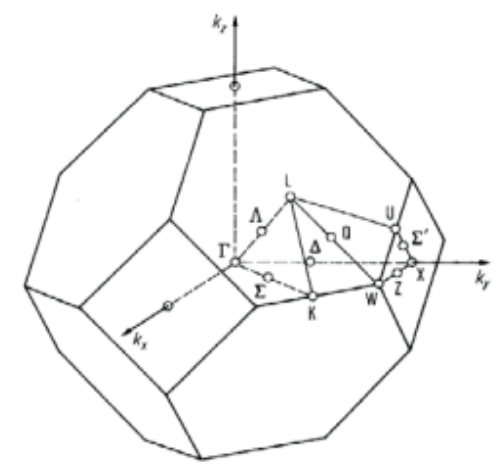

Figure 3. The Brillouin zone of the face-centered cubic lattice.

This is especially true for GdN. Various calculations have been undertaken on different magnetic phases (FM and AFM) with different schemes (LSDA and LSDA + U)of GdX (X $=\mathrm{N}, \mathrm{P}, \mathrm{As}, \mathrm{Sb}, \mathrm{Bi})$. The electronic structures, typical for rare-earth monopnictides, are presented in the discussion that follows.

Figure 4 shows the band structure of GdN in the FM state with and without on-site $4 \mathrm{f}$ Coulomb interactions. To construct the band structure in figure 4, we used an effective Hubbard $U_{\text {eff }}^{4 \mathrm{f}}=U-J=9.2 \mathrm{eV}$ for Gd compounds in the LSDA $+U$ scheme. As we will show later, this value gives much better agreement with experiment on the $4 \mathrm{f}$ energy levels than previous values did in the study of Gd metal or Gd compounds [142-144, 88, 89], i.e. $U=6.7$ and $J=0.7 \mathrm{eV}$.

As can be seen from figure 4(a), the LSDA $+U$ strategy has no significant impact on the whole LSDA band structure of GdN, except upon the energy levels of the occupied and unoccupied $4 \mathrm{f}$ states. In the pure LSDA scheme, the occupied $4 \mathrm{f}$ states are placed about 3.2 $\mathrm{eV}$ below $E_{\mathrm{F}}$, strongly hybridized with $\mathrm{N} 2 \mathrm{p}$ states, while the unoccupied $4 \mathrm{f}$ states are located about $2 \mathrm{eV}$ above $E_{\mathrm{F}}$, mixed with $\mathrm{Gd} 5 \mathrm{~d}$ states. There is no direct photoemission data from $\mathrm{GdN}$ single crystals, but experiments on $\mathrm{GdP}(\mathrm{As}, \mathrm{Sb}, \mathrm{Bi})$ show that the occupied and unoccupied $4 \mathrm{f}$ levels are situated $8-10$ and 5-6 eV below and above $E_{\mathrm{F}}$, respectively [59]. Furthermore, the x-ray photoelectron spectroscopy (XPS) experiments on GdN films show that the $\mathrm{Gd} 4 \mathrm{f}$ levels are $7.8 \mathrm{eV}$ below $E_{\mathrm{F}}$ [122]. Thus the LSDA calculation fails to give the correct $4 \mathrm{f}$ energy level binding energies. This situation is much improved by the LSDA $+U$ method (figure 4(b)). In fact, now the unoccupied 4f states remain around 6.6 $\mathrm{eV}$ above $E_{\mathrm{F}}$, and the occupied $4 \mathrm{f}$ states are $7.8 \mathrm{eV}$ below $E_{\mathrm{F}}$, in very good agreement with experiments. 


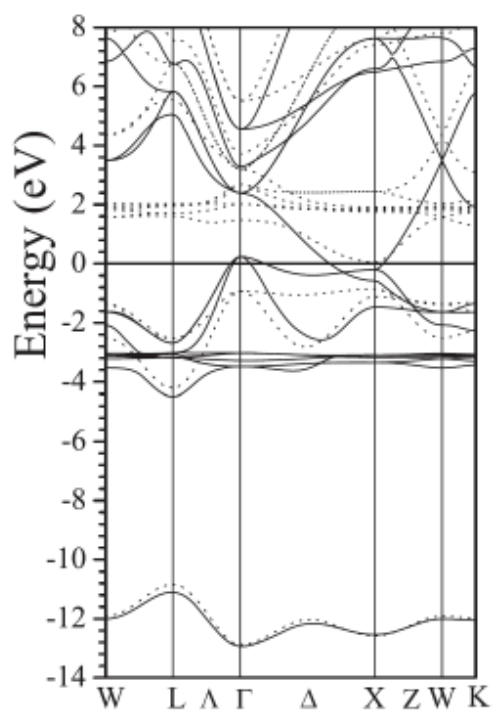

(a)

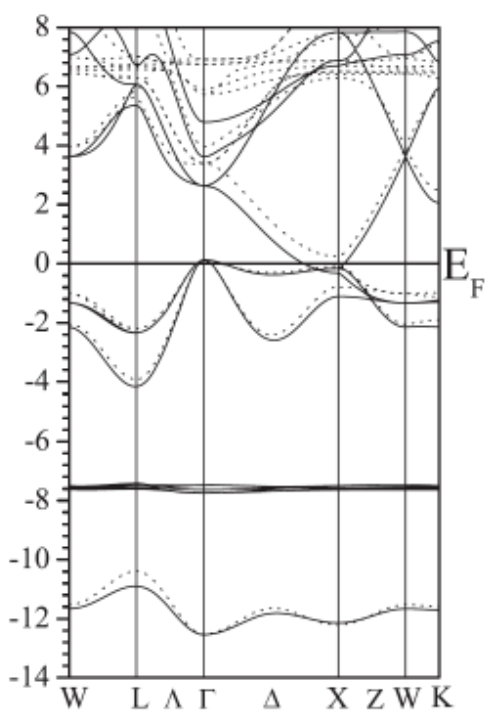

(b)

Figure 4. Electronic structure of ferromagnetic GdN for experimental lattice constants $(a=$ $4.974 \AA$ ): (a) LSDA, (b) LSDA $+U$ with $U_{\text {eff }}=9.2 \mathrm{eV}$. Solid and dotted lines represent spin majority and spin minority states, respectively.

Figure 4(b) is actually qualitatively representative and contains most of the typical features of the band structure of most rare-earth monopnictides. The pnictogen p-derived states (here N 2p) dominate the top of the valence bands. Precisely speaking, these pnictogen $p$ states are actually hybridized with rare-earth (here Gd) $5 d(6 s)$ states, which dominate the bottom of conduction band. This hybridization results in a hole pocket at the $\Gamma$ point and an electron pocket at the X point in metallic pnictides. The numbers of electrons and holes are the same [31]. Even if the rare-earth pnictides are determined to be semiconductors, the close proximity (and likely Fermi level crossing) of the unoccupied rare-earth $5 \mathrm{~d}$ states near the $\mathrm{X}$ point would make the energy gap indirect. A significant exchange splitting can be found in the band structure, and this is indeed the origin of the nominal half-metallicity in rare-earth monopnictides, as we will discuss in detail later.

The $l$-projected density of states (DOS) plot (figure 5) provides an even clearer picture of the elemental contributions to the electronic structure of GdN. As one can see, those top valence states right below the Fermi level are predominantly N 2p states. The Gd d states, however, also make a noticeable contribution. Note here that in our calculation both $5 \mathrm{~d}$ $\mathrm{e}_{\mathrm{g}}$ and $5 \mathrm{~d}_{2 \mathrm{~g}}$ states participate in the hybridization with $\mathrm{N} 2 \mathrm{p}$ states, though the contributions to the bottom of the conduction band(s) are dominated by $5 \mathrm{~d} \mathrm{t}_{2 \mathrm{~g}}$ states. Not only do the calculated $4 \mathrm{f}$ levels agree well with experiment, but the $\mathrm{Gd} 5 \mathrm{p}$ states are located at the same binding energy position (about $21 \mathrm{eV}$ below $E_{\mathrm{F}}$ ) found in experiment [122]. The 1.8 $\mathrm{eV}$ separation between the Gd 5p majority and minority states is caused by the exchange splitting. We would like to point out that the Gd $5 \mathrm{~d}-\mathrm{X} 2 \mathrm{p}$ hybridization is important in establishing the physical properties of $\mathrm{GdX}$. This hybridization results in magnetic moments on pnictogen sites and is responsible for the intriguing magnetic orderings and transport properties. 


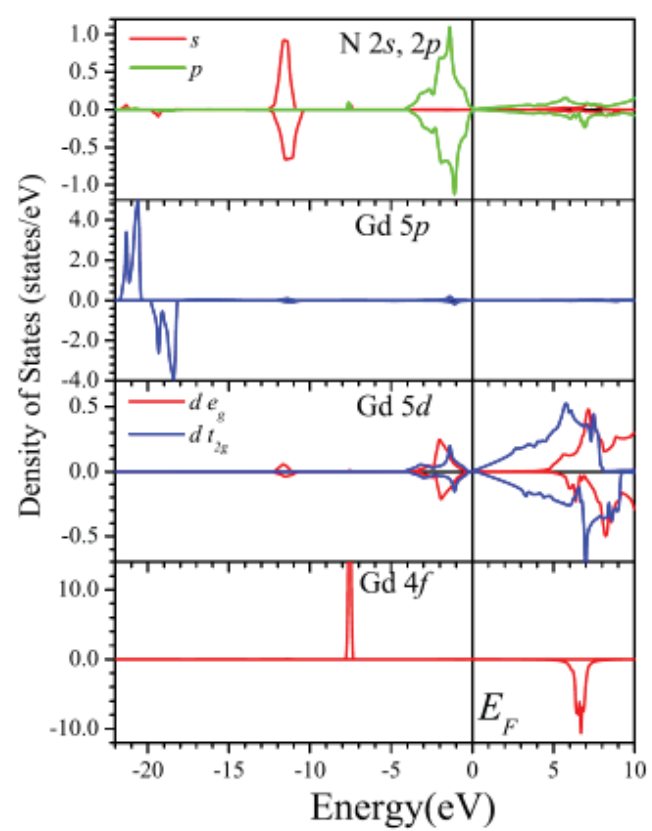

Figure 5. The $l$-projected DOS of GdN. Spin minority states are of negative values.

Band structures for other Gd monopnictides in the ferromagnetic state, using LSDA $+U$, are shown in figure 6 . Note that the magnetic ground states of these compounds are AFM-II type. We can consider these structures as the saturated limit of the paramagnetic states in a magnetic field [36].

The band structures seen for the rare-earth pnictides from GdN to GdBi are quite similar, except that for GdSb and GdBi there are no gaps between the $\mathrm{Gd} 5 \mathrm{~d}$ states and pnictogen $\mathrm{p}$ states at the $\Gamma$ point. We find that, with a single universal $U_{\text {eff }}^{4 \mathrm{f}}$ the $\mathrm{f}$ levels in these compounds, both occupied and unoccupied, are all in excellent agreement with experimental observations $[59,122]$, vindicating the application of the LSDA $+U$ scheme. This is clearly shown in the XPS and x-ray bremsstrahlung isochromat spectroscopy (X-BIS) spectra of GdX (figure 7). There exists a monotonic energy down shift from GdN to GdBi [59], which is clearly represented in the LSDA + $U$ band structures of GdX.

From the LSDA $+U$ calculations, all the Gd monopnictides are seen to be semi-metallic except for GdN, which is nominally half-metallic with a half-metallic gap about $0.5-0.6$ eV. Experimentally, the GdP (As, Sb, Bi) pnictides are found to be metallic [58]. For GdN, as noted previously, the experimental temperature-dependent resistivity of the GdN film [126] shows a picture (figure 8) that differs from other GdX compounds (figure 9,[58]). The high-temperature behavior of the resistivity of GdN is more like that of a semiconductor, apparently different from other Gd monopnictides. The resistivity peak around the transition temperature can be explained by the long-range magnetic ordering which significantly decreases the spin-dependent scattering of charge carriers, well below the transition temperature. The broad range of this peak may be attributed to the imperfection periodicity or the Kondo-lattice model $[145,146]$. Though the resistivity at very low temperature is not huge $(5 \Omega \mathrm{cm})$, it is again still more like a semiconductor.

If $\mathrm{GdN}$ is really a semiconductor, with an optical gap about $1.5-2 \mathrm{eV}$ [126], then it means that there still is something important missing in the ' $a b$ initio' calculations. Lam- 


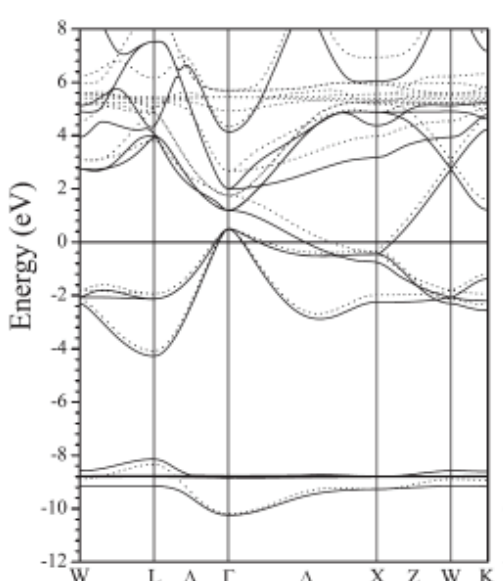

(a)

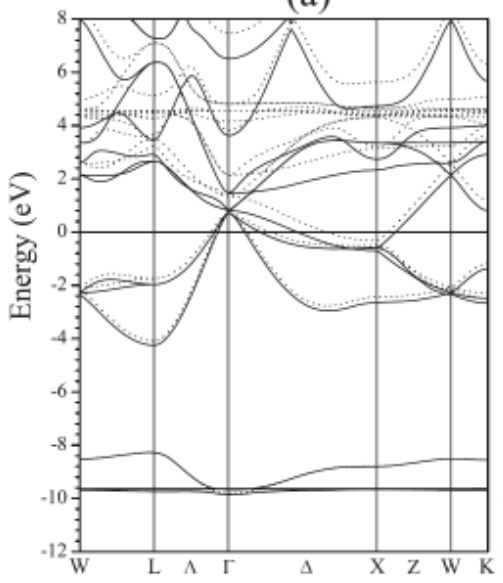

(c)

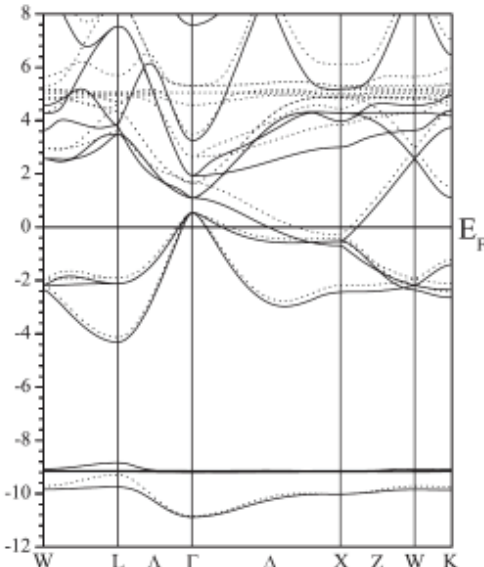

(b)

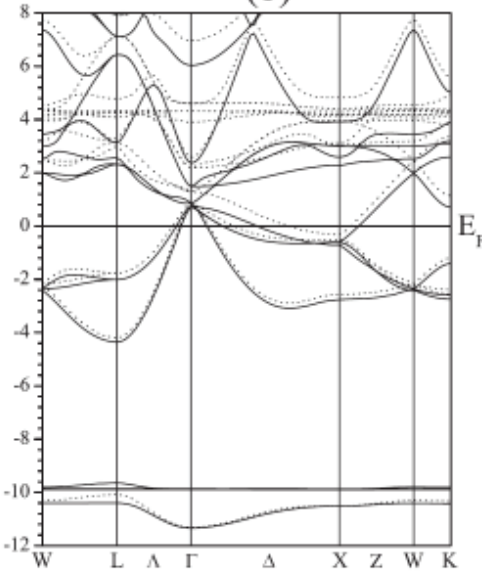

(d)

Figure 6. Electronic structure (LSDA $+U$ with $\left.U_{\text {eff }}=9.2 \mathrm{eV}\right)$ of (a) GdP $\left(a_{\exp }=5.709 \AA\right)$, (b) $\operatorname{GdAs}\left(a_{\exp }=5.864 \AA\right),(\mathrm{c}) \mathrm{GdSb}\left(a_{\exp }=6.219 \AA\right),(\mathrm{d}) \mathrm{GdBi}\left(a_{\exp }=6.295 \AA\right)$ in the ferromagnetic phase.

brecht [103], Ghosh et al [92], Larson and Lambrecht [93] used different approaches to shift the Gd 5d bands and managed to make GdN semiconducting. These empirical treatments of the theory do not give the correct positions of the $\mathrm{Gd} 4 \mathrm{f}$ levels. Apparently, more effort is needed to fill the persistent gap between theory and experiment.

Since the ground states of $\mathrm{GdX}(\mathrm{X}=\mathrm{P}, \mathrm{As}, \mathrm{Sb}, \mathrm{Bi})$ are all of AFM-II type (see figure 2(b)), it is interesting to compare the difference between the AFM and FM electronic structures. Figure 10 shows both AFM and FM bands of GdP with the same rhombohedral cell (where the Brillouin zone is shown in figure 11) and adopting the experimental lattice constants. As we can see, the AFM bands show an average effect of the FM spin majority and minority bands, and this leads to a small reduction in total energy. The essential feature of the electronic structure, however, remains the same.

From the above analysis, apparently GdN is on the border between semiconductor and semimetal states. Thus the GdN physical properties are very sensitive to non-stoichiometry, impurities, pressure, temperature, magnetic field, etc. In addition, exotic phenom- 


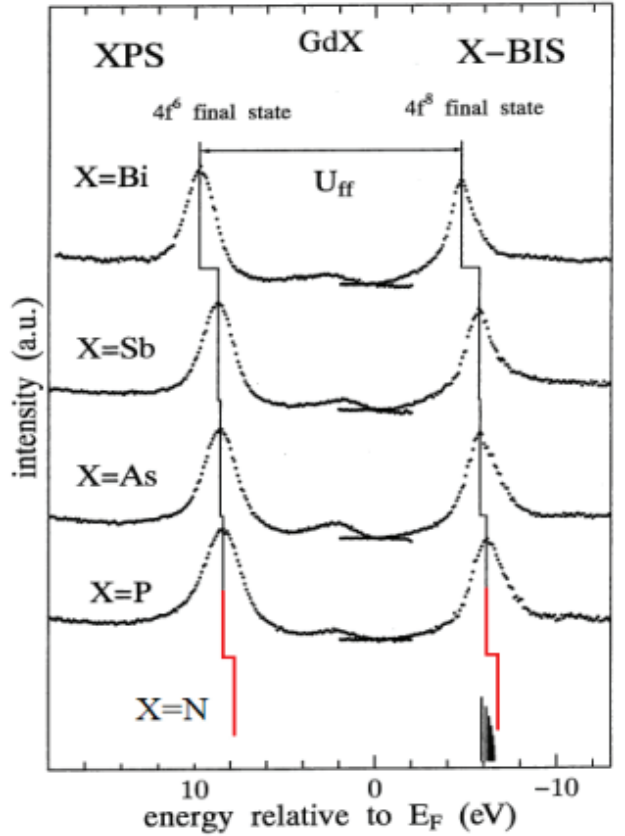

Figure 7. The XPS and X-BIS spectra of $\operatorname{GdX}(X=P, A s, S b, B i)$ (from [59]). Vertical line indicating the occupied $4 \mathrm{f}$ level of GdN is from [122], the unoccupied GdN $4 \mathrm{f}$ level is calculated using the $U_{\mathrm{ff}}$ indicated in the figure.

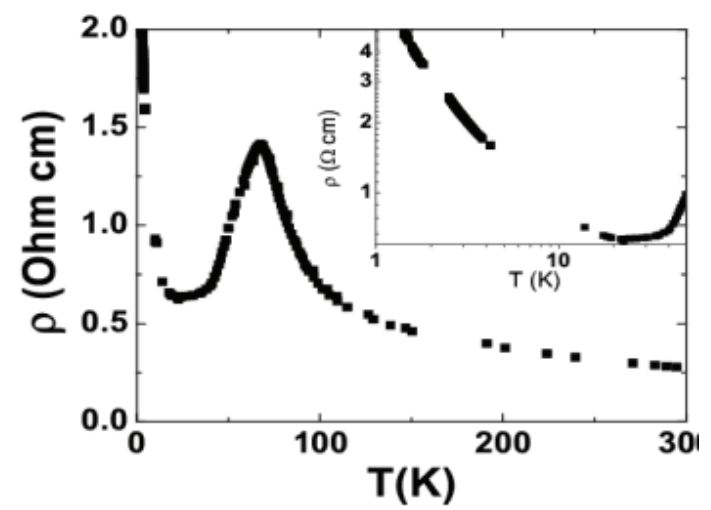

Figure 8. Temperature-dependent resistivity of a $200 \mathrm{~nm}$ thick GdN film. The pronounced peak at $68 \mathrm{~K}$ corresponds to the measured Curie temperature of the film. The inset shows the low-temperature behavior of the resistivity (from [126]).

ena, such as electron-hole liquids [147], may occur in the region between semiconductor and semimetal states. Again, if it is true that GdN is a semiconductor and GdP is a semimetal, then most probably there should exist some GdN-GdP alloys that would be nominally half-metallic.

\subsection{Eu monopnictides}

In the europium pnictide family only EuN and EuP crystallize with fcc structure. According to Hund's rules [148], the ground state of $\mathrm{Eu}^{3+}\left(4 \mathrm{f}^{6}\right)$ is ${ }^{7} \mathrm{~F}_{0}$, i.e. $S=3, L=3, J=0$. Thus 


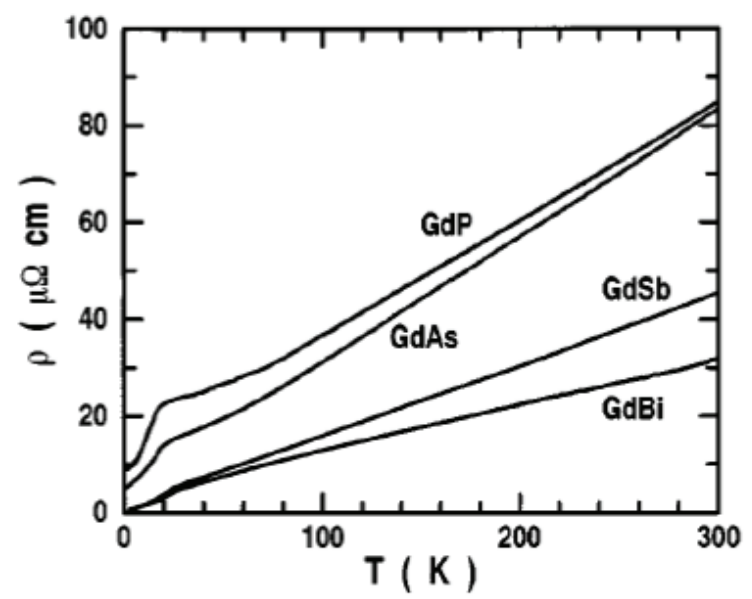

Figure 9. Temperature-dependent resistivity of stoichiometric $\mathrm{GdX}(\mathrm{X}=\mathrm{P}, \mathrm{As}, \mathrm{Sb}$, Bi) single crystals measured at zero field (from [58]).

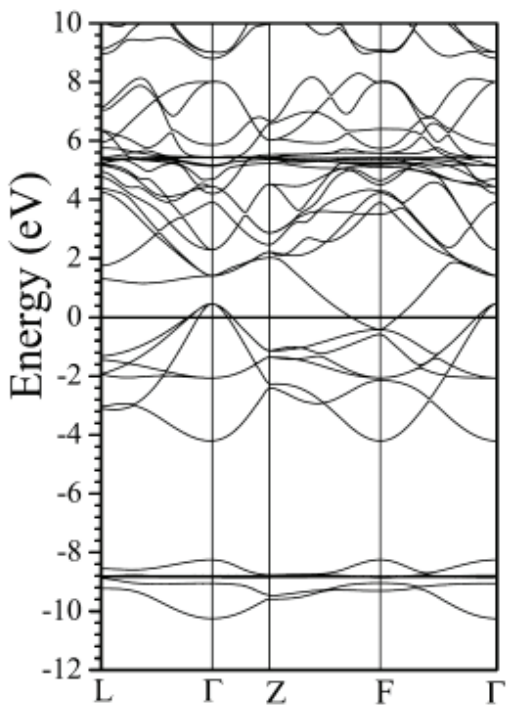

(a)

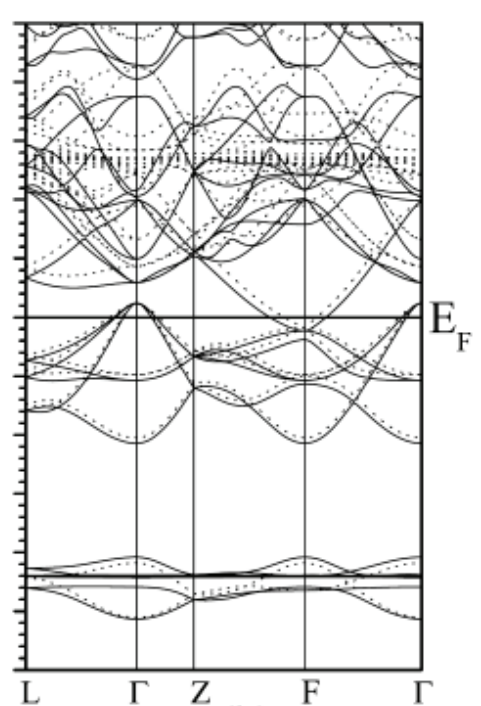

(b)

Figure 10. Electronic structure of GdP with rhombohedral cell: (a) antiferromagnetic phase (AFM-II), (b) ferromagnetic phase. Note that in antiferromagnetic phase there is no difference between the spin majority and minority states.

isolated $\mathrm{Eu}^{3+}$ is supposed to be non-magnetic. In the crystalline environment, however, the orbital moment generally will be suppressed by the crystal field; thus net magnetic moments may exist in $\operatorname{EuX}(X=N, P)$. In addition, as in the case of Gd monopnictides, there could be induced magnetic moments on pnictogen atoms. Taking into account the spin-orbit coupling, bulk $\mathrm{Eu}^{3+}$ could exhibit significantly different magnetic properties from that of isolated $\mathrm{Eu}^{3+}$.

Experimental studies on the electronic structure of the $\operatorname{EuX}(X=N, P)$ compounds are very rare. Theoretical calculations are also limited. So far there have only been two published calculations on europium monopnictides, to our knowledge. As previously noted, Horne et al reported EuN to be half-metallic based on the SIC-LSDA method [71] and was confirmed by Johannes and Pickett [91] using the LSDA $+U$ scheme. 


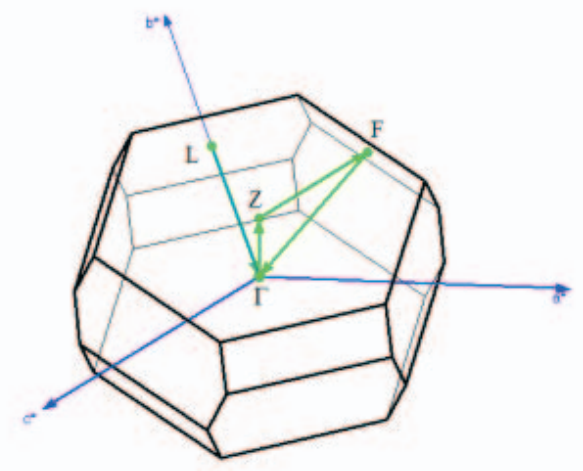

Figure 11. The Brillouin zone of the rhombohedral lattice.

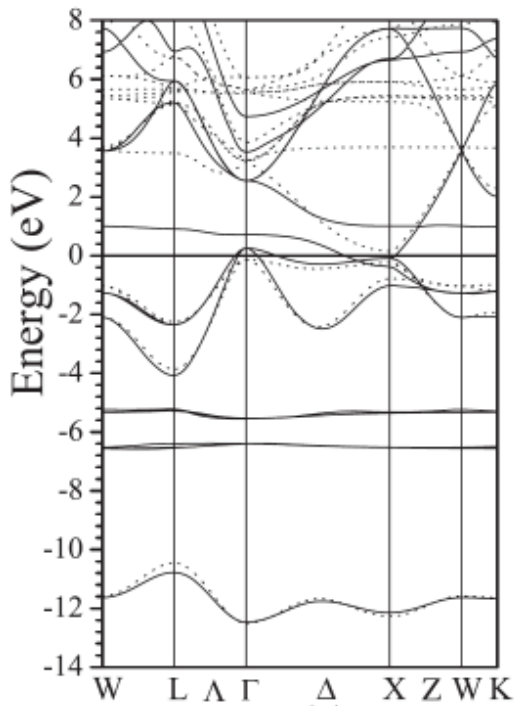

(a)

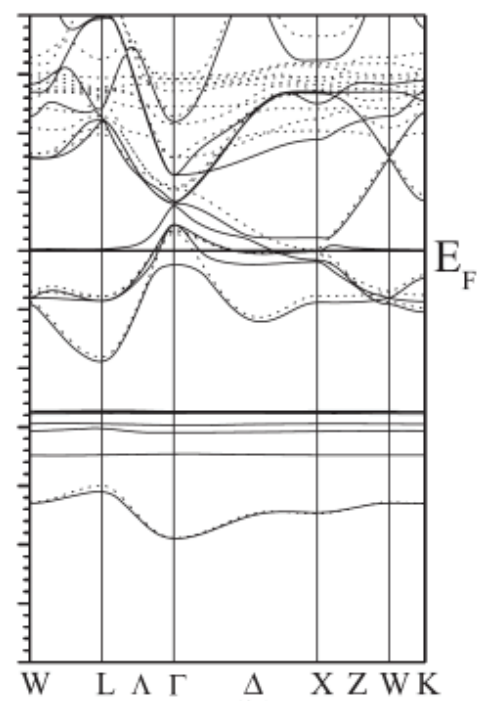

(b)

Figure 12. Electronic structure (LSDA $+U$ with $\left.U_{\text {eff }}=7 \mathrm{eV}\right)$ of (a) $\operatorname{EuN}\left(a_{\exp }=5.017 \AA\right)$ and (b) $\operatorname{EuP}\left(a_{\exp }=5.756 \AA\right)$ in ferromagnetic phases.

Following Johannes and Pickett [91], using the LSDA $+U$ approach with $U$ and $J$ set as 8 and $1 \mathrm{eV}$ respectively, we have obtained similar band structures for EuN and EuP, as shown in figure 12. The spin-orbital effects were not considered in these calculations and will be discussed later. Johannes and Pickett's calculations showed that the ground states of EuN and EuP are FM and AFM-II, respectively. Here we only present results of the FM states of two compounds for comparison. The difference between the FM and AFM states of EuP is very similar to the case of GdP (figure 10).

As we can see from figure 12, the band structures of Eu monopnictides are similar to those of Gd monopnictides (figures 4 and 6). The major differences arise from the unoccupied $4 \mathrm{f}$ majority states. In EuN, this state is about $1 \mathrm{eV}$ above $E_{\mathrm{F}}$, whereas in EuP this state crosses the Fermi level. In both cases, the unoccupied 4f states are strongly hybridized with Eu $5 \mathrm{~d}$ and pnictogen p states. Figure 12(a) shows that, in this conventional LSDA + $U$ band calculation, EuN is half-metallic with a $0.5 \mathrm{eV}$ gap in the minority spin channel. Other Eu monopnictides are semimetallic, like EuP, as illustrated in figure 12(b). 


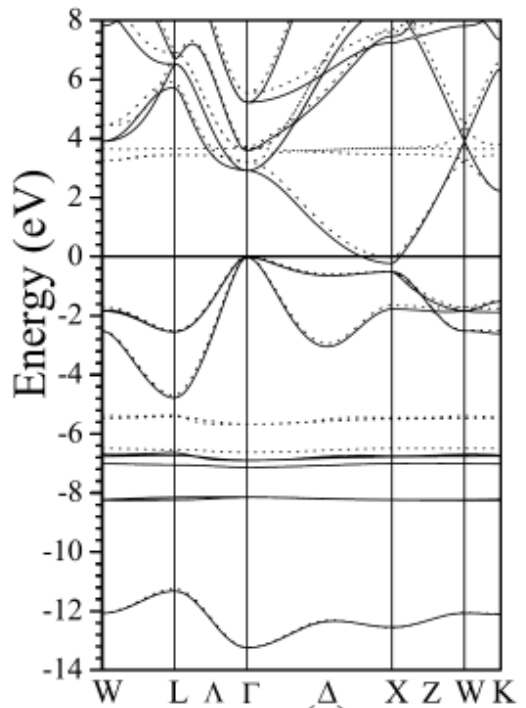

(a)

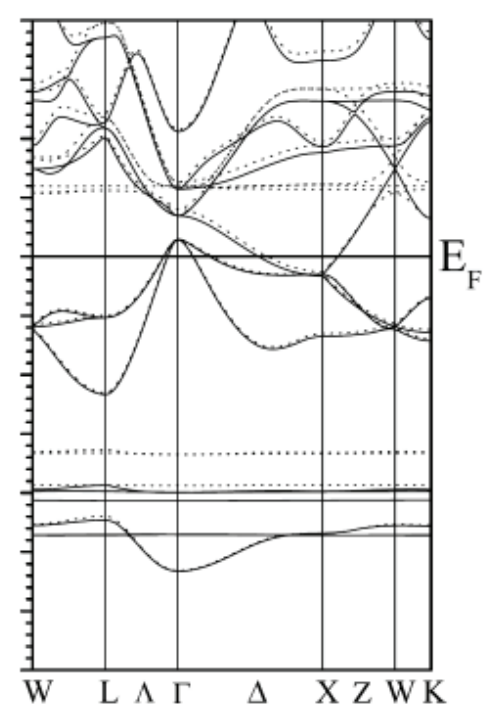

(b)

Figure 13. Electronic structure (LSDA $+U$ with $\left.U_{\text {eff }}=7.85 \mathrm{eV}\right)$ of (a) $\operatorname{ErN}\left(a_{\exp }=4.835 \AA\right)$, (b) $\operatorname{ErP}\left(a_{\exp }=5.595 \AA\right)$ in ferromagnetic phases.

\subsection{Er monopnictides}

$\mathrm{Eu}^{3+}$ represents another type of rare-earth ion, which has more than a half-filled $4 \mathrm{f}$ electron shell. This provides a contrast with Gd (with a half-filled $4 \mathrm{f}$ electron shell) and $\mathrm{Eu}$ (with a less than half-filled $4 \mathrm{f}$ electron shell). The ground state of isolated $\mathrm{Eu}^{3+}\left(4 \mathrm{f}^{11}\right)$ is ${ }^{4} \mathrm{I}_{15 / 2}$, i.e. $S=3 / 2, L=6, J=15 / 2$. Only ErAs has been studied extensively experimentally [50, 51, 84], whereas theoretical studies of all of the Er monopnictides have been undertaken [35, 36, 70, 84, 85, 87]. Except for ErN, all Er monopnictides have AFM ground states and have similar electronic structures and physical properties, nonetheless, here we present our LSDA $+U$ band calculations for ErN and ErP in the ferromagnetic state (figure 13). Following Komesu et al [84], $U$ and $J$ are chosen to be 8.6 and $0.75 \mathrm{eV}$, respectively.

In the Er monopnictides, all the $4 \mathrm{f}$ majority states and four of the seven $4 \mathrm{f}$ minority are occupied. Because of the Coulomb repulsion, the unoccupied $4 \mathrm{f}$ states reside 3.2-3.6 eV above $E_{\mathrm{F}}$ in ErN. A zero half-metallic gap is found for ErN in the LSDA $+U$ band calculations, whereas ErP is semimetallic in both spin channels with the $4 \mathrm{f}$ bands shifted down about $1 \mathrm{eV}$ (figure 13).

\section{Half-metallicity and other interesting phenomena}

\subsection{Origins of half-metallicity}

In band calculations, half-metallicity will not occur if there is no exchange splitting. While an exchange splitting is a necessary condition for half-metallicity, exchange splitting alone is not sufficient, even in the rare-earth monopnictides. The exchange splitting must be large enough to form a gap between the unoccupied and occupied states in one spin channel and at the same time the other spin channel should be partially occupied. Thus the formation of halfmetallicity strongly depends on the magnitude of exchange splitting as well as the details of the electronic structure. For a perfect ionic or covalent crystal, the exchange split- 


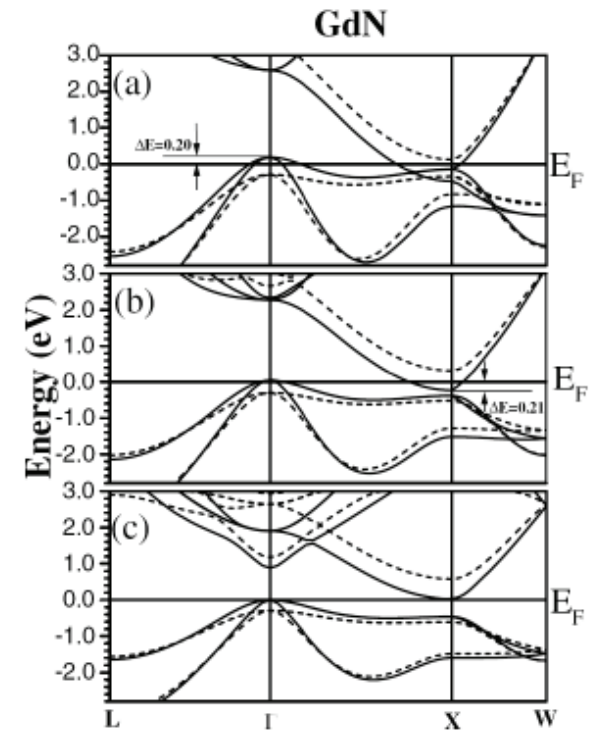

Figure 14. The LSDA $+U\left(U_{\text {eff }}=6.0 \mathrm{eV}\right)$ band structure of $\mathrm{GdN}$ in the vicinity of the Fermi energy for three volumes: (a) at the calculated equilibrium lattice parameter $a=4.92 \AA$, (b) at the lattice parameter increased by $5 \%(a=5.16 \AA)$, (c) at the lattice parameter increased by $14 \%(a=5.63 \AA)$. Solid and dotted lines represent spin majority and spin minority states, respectively. The change of conducting properties is indicated by the change of energy difference between the top (bottom) of the hole (electron) pockets and the Fermi energy (from [88]).

ting is zero due to the fully occupied outermost electron shell, which also makes the system insulating. For a partially occupied electron shell, the exchange splitting is generally around $0.1-1.5 \mathrm{eV}$ in most elements and compounds, whereas the width of unoccupied bands is generally above $3-4 \mathrm{eV}$. As a consequence half-metallic materials are rather rare.

In some sense, the band structure of rare-earth monopnictides resembles another class of nominally half-metallic materials: the full-Heusler alloys [149]. In the band structure there is a hole pocket around the $\Gamma$ point and a electron pocket around the $\mathrm{X}$ point (figure 4). The spin exchange splitting is of opposite sign for the top valence and bottom conduction bands, i.e. the exchange splitting pushes the majority states to the top of the valence band (at the $\Gamma$ point) and simultaneously pushes down the minority states (at the X point). Thus, under some conditions, a gap forms in the spin-down channel in some calculated band structures.

Rare-earth systems are known to exhibit band- and wavevector-dependent exchange splitting $[9,11,150,151]$. Taking GdN as an example, we see that the Gd 5d states are polarized by the $4 \mathrm{f}$ majority states, due to exchange interactions and $4 \mathrm{f}-5 \mathrm{~d}$ orthogonality. This means that the Gd $5 \mathrm{~d}$ majority spin states, which dominate the bottom conduction bands, are shifted to greater binding energies. At the same time, the $\mathrm{Gd} 5 \mathrm{~d}$ states are hybridized with N $2 p$ states. This hybridization shifts up the N 2p majority spin states, resulting in the exchange splitting at the top valence bands, which mainly consist of the $\mathrm{N} 2 \mathrm{p}$ electrons. This shift in weight from Gd $5 \mathrm{~d}$ to $\mathrm{N} 2 \mathrm{p}$ does result in a wavevector-dependent exchange splitting. We note that the exchange splitting is also Hubbard $U$ dependent, as can be seen from the differences between figures 4(b) and 14(a). This is because the larger $U$ of the $f$ states means fewer $\mathrm{f}-\mathrm{d}$ interactions, and thus a smaller exchange splitting.

Another by-product of the above Gd $5 \mathrm{~d}-\mathrm{N} 2 \mathrm{p}$ hybridization is the induced magnetic moments on nitrogen atoms. Actually, the induced magnetic moment on the pnictogen 
atom is a typical feature of rare-earth monopnictides. This was first pointed out by Petukhov et al [36] and has been confirmed by recent experiments [122]. This induced pnictogen magnetic moment is antiparallel to that of the rare-earth atoms, with a magnitude in the range of $0.05-0.14 \mu_{\mathrm{B}}$. Such an induced antiparallel magnetic moment on the nonmagnetic atom is also found in magnetic interface structures, for example $\mathrm{Co} / \mathrm{SrTiO}_{3}[152$, 153] and Fe/BTO [154], in which the non-magnetic Ti atom has a negative magnetic moment due to the Ti $3 \mathrm{~d}-\mathrm{Fe} 3 \mathrm{~d}$ hybridization.

\subsection{Metal-insulator transitions}

We can see from table 1, figure 4 and figure 6 that the metallicity of rare-earth monopnictides gradually increases from $\mathrm{N}$ to $\mathrm{Bi}$. Since experimentally most rare-earth nitrides are semiconductors, we can reasonably expect a metal-insulator transition in some rare-earth pnictide compounds or their alloys, due to impurity doping, temperature, pressure or even magnetic field.

Duan et al [88] studied both the volume and lattice strain effect on the electronic properties of GdN using $U_{\text {eff }}^{4 \mathrm{f}}=6 \mathrm{eV}$. The results are shown in figure 14. It is evident that, at larger volumes, the electron (hole) pockets become substantially shallower, meaning that the bottom (top) of the band approaches the Fermi energy and the area of the Fermi surface decreases. Both carrier density and carrier mobility decreases with an increase of the cell volume. Though it is hard to realize such a dramatic volume change in practice, the trend should be correct in the regimes that prove to be experimentally accessible. This transition is close to resembling a Mott metal-insulator transition, and such models [155] may be used to explain the gap in $\mathrm{GdN}$ found experimentally.

We note that there is another mechanism of metal-semiconductor transition, i.e. through $\mathrm{d}-\mathrm{f}$ or $\mathrm{s}-\mathrm{f}$ hybridization. In the famous Falicov-Kimball model [156], the single-electron states consist of extended Bloch functions and localized states centered on the metallic ions in the crystal. At low temperatures, the quasiparticle excitations are either localized holes or itinerant electrons. The electron-hole interaction may be responsible for the semiconductor- metal phase transition. The same spirit is presented in the electron-hole liquid theory [147], which is used to explain the ground state of ScN. Using a novel many-body theory and Kondo lattice model ( $\mathrm{s}-\mathrm{f}$ model), Kreissl and Nolting predicted that the decreasing magnetic order induces a transition from half-metallic to semiconducting behavior in $\mathrm{EuB}_{6}$ [157]. If this can be confirmed experimentally, then similar examples should be found among the rare-earth monopnictides.

\subsection{Magnetic ordering}

The magnetic properties are closely related to the electronic and transport properties in rare-earth monopnictides. For instance, a dramatic change of electric resistance has been found near $T_{\mathrm{c}}$ in $\operatorname{GdN}[122,126]$. Despite their rather simple fcc structure, the magnetic orderings of the rare-earth pnictides are very complicated [22, 23, 39, 54]. Based on the experience with rare-earth metals $[9,144]$, it is generally believed that there exists an indirect RKKY exchange interaction in the rare-earth pnictides. In 1997, Li et al estimated the exchange parameters of GdX from experimental data [60]. They attributed the sign change of exchange parameters to RKKY oscillations. Several groups believe that the superexchange interaction should also exist, and may dominate these compounds [88, 89, 91].

Duan et al $[88,89]$ proposed a systematic way to study the magnetic ordering in the rare-earth pnictide fcc structures. The basic strategy is to first deduce the exchange interac- 

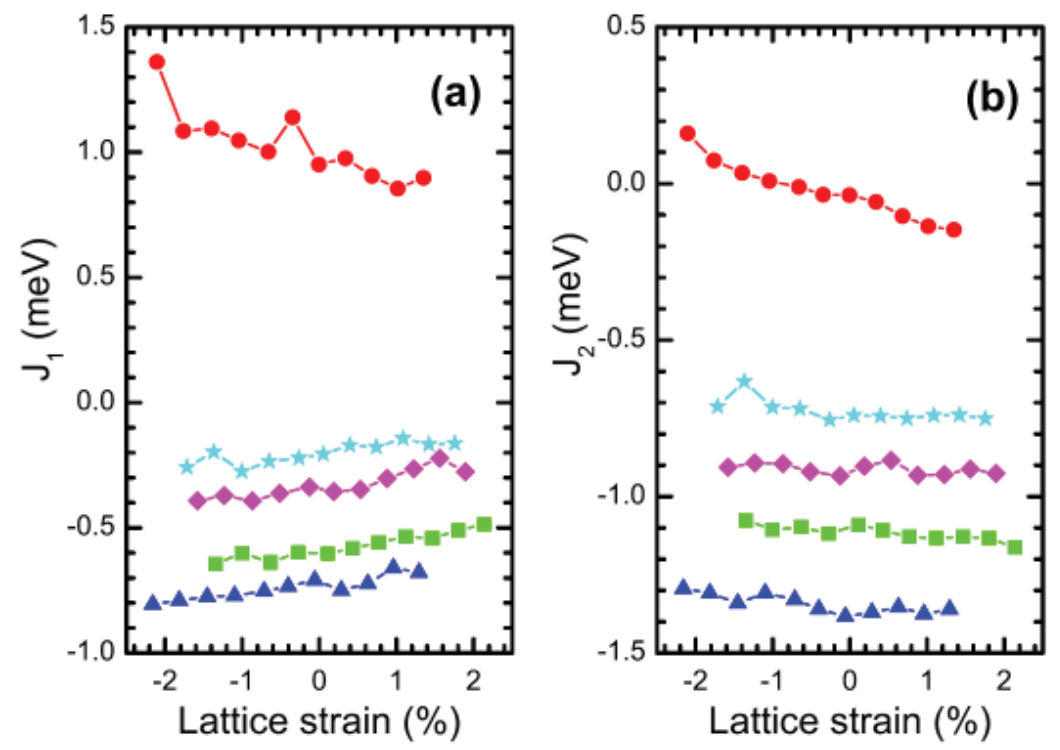

Figure 15. Calculated exchange parameters $J_{1}$ (a) and $J_{2}$ (b) for Gd pnictides as a function of the lattice strain: GdN (circles), GdP (stars), GdAs (diamonds), GdSb (squares), GdBi (triangles). The lattice strain is defined by the relative deviation of the lattice constant from the theoretical equilibrium lattice constant (from [89]).

tion parameters of GdX compounds by fitting the first-principles total energies of different magnetic configurations to those computed within the Heisenberg model,

$$
H=-\sum_{n=1,2,3} J_{n} \sum_{i>j}^{n \text {th NN }} \mathbf{S}_{i} \cdot \mathbf{S}_{j} .
$$

Here $\mathrm{S}_{i}$ is the unit vector in the direction of the magnetic moment at the $i$ th lattice site, $J_{n}$ is the exchange parameter between the $n$th nearest-neighbor (NN) magnetic atoms, and we limit our consideration to third-NN interactions. In this case the differences between the energy of the three AFM states $E_{n}(n=\mathrm{I}$, II, III) shown in figure 1 and the energy of the FM state $E_{\mathrm{FM}}$ are

$$
\begin{aligned}
& \Delta E_{\mathrm{I}}=E_{\mathrm{I}}-E_{\mathrm{FM}}=8 J_{1}+16 J_{3}, \\
& \Delta E_{\mathrm{II}}=E_{\mathrm{II}}-E_{\mathrm{FM}}=6 J_{1}+6 J_{2}+12 J_{3}, \\
& \Delta E_{\mathrm{III}}=E_{\mathrm{III}}-E_{\mathrm{FM}}=8 J_{1}+2 J_{2}+8 J_{3} .
\end{aligned}
$$

The so-derived exchange parameters are then put into Monte Carlo simulations to calculate the transition temperatures of the compound. Results for GdX are shown in table 2.

The lattice constant dependences of the exchange parameters $J_{1}$ and $J_{2}$ are also obtained (figure 15). From the analysis of these data, Duan et al [89] proposed an expression for the superexchange interaction:

$$
J_{2}^{\text {super }}=-n_{\mathrm{d}} \frac{t_{\mathrm{pd}}^{4}}{\Delta^{3}},
$$

where $\Delta$ is the energy difference between the rare-earth $5 \mathrm{~d}$ orbital and the outmost $\mathrm{p}$ state of the pnictogen ion, $n_{\mathrm{d}}$ is the induced $\mathrm{d}$ moment on the rare-earth atom due to atomic $4 \mathrm{f}-5 \mathrm{~d}$ exchange interactions, and the hopping parameter $t_{\mathrm{pd}}$ can be evaluated according to 


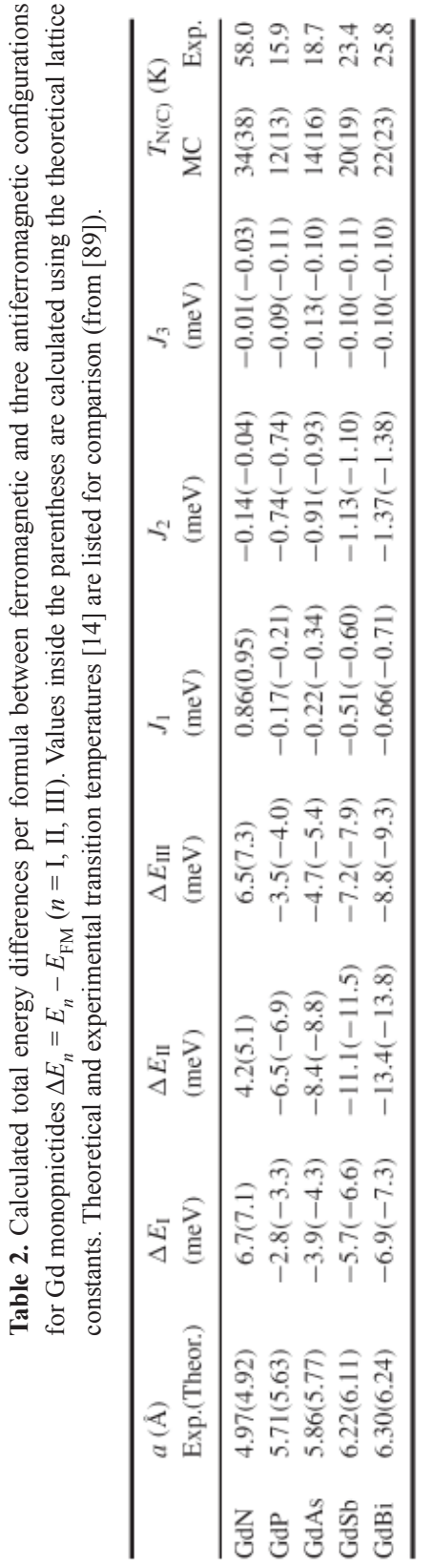


Harrison [158]. We should note here that the evaluation of equation (11) is quite empirical, since accurate determinations of $\Delta$ and other parameters are not available. Nevertheless, the estimated superexchange interactions in GdX are found to be AFM and have the same magnitude as the total effective magnetic interactions. These AFM superexchange interactions are strengthened when the size of the pnictogen atoms increases, and they eventually dominate the magnetic interactions in rare-earth pnictides. This picture agrees well with experimental observations [60]. Using similar technique, Larson and Lambrecht [93] also obtained the exchange parameters of GdX in good agreement with experiment.

Duan et al also investigated the magnetic dipole interactions and magnetic anisotropy effects in rare-earth monopnictides. They found that dipole-dipole interactions could play an important role in determining the magnetic behavior of the rare-earth compounds, and are especially instrumental in the magnetic anisotropy, but they cannot solely account for stabilizing the magnetic ordering in an fcc structure [51].

It should be pointed out that although the calculated Curie temperatures, using theoretical exchange coupling parameters within the Heisenberg model, agree reasonably well with experiment, it is still difficult to uniquely attribute the mechanism of exchange interactions in these compounds to the RKKY or superexchange mechanisms. The relative contribution from RKKY interaction varies considerably due to the change in metallicity across the monopnictides. For example, if the system is insulating, the RKKY interactions should vanish. Therefore, in addition to the RKKY exchange and superexchange contributions, other exchange coupling mechanisms are possible in the rare-earth monopnictides, such as higher order exchange interactions [37], direct exchange, and double exchange interactions. Actually, there is experimental evidence that direct exchange coupling could be responsible for the higher Curie temperature found in insulating GdN films [126].

\subsection{Spin-orbit coupling}

Spin-orbit coupling is important for correctly deriving the band structure and many other properties, e.g. magneto-crystalline anisotropy, of compounds containing heavier elements like rare-earths. For Gd ions, the spin-orbit term $\xi \sigma \cdot L$ (here $\sigma$ and $L$ are spin and orbital angular momentum operators, respectively) may be ignored since the $4 \mathrm{f}$ shells are exactly half occupied. For other rare-earth ions with non-zero total orbital angular momentum, the spin-orbit term may be significant. There are very few ab initio calculations on the rareearth pnictides involving spin-orbit coupling. Duan et al studied the spin-orbit effect in ErAs [85], and Johannes and Pickett also considered this effect in their studies on EuN and EuP [91]. Leuenberger et al mentioned the presence of an important spin-orbit interaction in the final $\mathrm{N}$ p states of $\mathrm{GdN}$ [122].

Figure 16 presents the theoretical band structure of ErAs that takes into account both the Hubbard $U$ term and the spin-orbit interaction. In this calculation, the inclusion of spin- orbit interaction is realized by a fully relativistic approach for the core electrons and a second variational method for the valence states [159]. In contrast to the LSDA $+U$ result [84], the occupied $4 \mathrm{f}$ bands are split further due to spin-orbit coupling, leading to better agreement with experiments [85].

Spin-orbit coupling is at the heart of magneto-optical effects such as Kerr and Faraday polarization rotation. $\mathrm{CeSb}$ has been claimed to have a $90^{\circ} \mathrm{Kerr}$ rotation [160]. While the intrinsic value of the Kerr rotation of $\mathrm{CeSb}$ is still debated, agreement exists that $\mathrm{CeSb}$ has possibly the largest Kerr rotation, possibly the largest. A series of calculations of the magneto-optical properties of the rare-earth monopnictides reproduced experimental results with varying success $[83,92,161]$. 


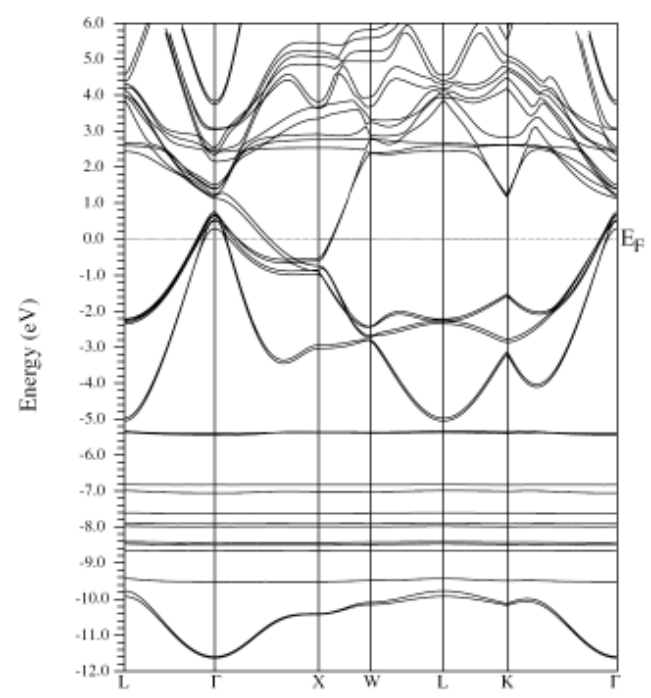

Figure 16. The calculated LSDA $+U+$ SO band structure of bulk ErAs. In the spin-orbit calculation, spin-up and spin-down states are mixed (from [85]).

\section{The future possibilities}

While the rare-earth monopnictides have the simplest structure of the rare-earth compounds, substantive changes can be easily undertaken by altering the constituents. Actually, by mixing rare-earth pnictides in different ratios, we can tune the physical properties, for example $T_{\mathrm{c}}$, carrier density, and resistance, of rare-earth pnictides to desired values [24, $38,43,47,35,164]$. Impurity doping, and thus non-stoichiometry, also has a significant influence on the properties of rare-earth pnictides. These subjects are too complicated and are beyond the scope of this review. We just want to mention that there are many choices for improving the physical, magnetic and transport properties of rare-earth pnictides.

For binary rare-earth pnictide compounds, there are various types with different structures. For example, there are at least five structure types in the $\mathrm{RX}_{2}$ phase, i.e. $\mathrm{LaP}_{2}, \mathrm{NdAs}_{2}$, $\mathrm{SmSb}_{2}, \mathrm{HoSb}_{2}, \mathrm{ZrSi}_{2}$ [39]. Of particular interest, we mention the anti- $\mathrm{Th}_{3} \mathrm{P}_{4}$-type pnictides, which have attracted much interest recently $[162,163]$. Some of the anti- $\mathrm{Th}_{3} \mathrm{P}_{4}$-type pnictides demonstrate very large values for $T_{c}$, as noted below. For ternary compounds, the situation is much more complicated [39]. Active experimental studies are being carried out on these systems [164-166].

\subsection{Possible half-metallic antiferromagnets}

In 1995, Van Leuken and de Groot proposed another type of half-metallic material, i.e. the half-metallic antiferromagnet [167]. This material is calculated to be $100 \%$ spin polarized at the Fermi surface but has zero net magnetization, and thus could be used as a tip material in spin-polarized scanning tunneling microscopy. There are several theoretical predictions concerning the existence of the half-metallic antiferromagnet [167-170], but none of them has been confirmed experimentally.

Half-metallic antiferromagnets cannot be found with the simple pure rare-earth monopnictides, because the metallic spin channels are present in the two AFM sites of such materials. However, a mixed antiferromagnet such as EuO-GdN with $\mathrm{Eu}^{2+}$ and $\mathrm{Gd}^{3+}$ 
aligned antiparallel, could have electronic structure suitable for half-metallic behavior. If this prediction is verified, then this material would be a half-metallic antiferromagnet with the simplest structure (double fcc).

\subsection{Enhancement of the transition temperatures and potential device applications}

The rare-earth monopnictide compounds have generally rather low transition temperatures. GdN has the largest $T_{\mathrm{c}}(58-68 \mathrm{~K})$, but it is still far below room temperature. How to improve the $T_{\mathrm{c}}$ in these compounds is of interest.

In 1971, Kuznietz reported the effect of anion substitution in GdN and UN [171]. It was found that carbon substitution can significantly improve $T_{\mathrm{c}}$, while $\mathrm{O}$ substitution works the other way. An example is $\mathrm{GdN}_{0.88} \mathrm{C}_{0.12}\left(\mathrm{Gd}_{4} \mathrm{~N}_{3.5} \mathrm{C}_{0.5}\right.$ in [38]), whose $T_{\mathrm{c}}$ was reported to be as high as $190 \mathrm{~K}$. This can be explained by the reduction of $\mathrm{Gd}-\mathrm{Gd}$ distance, which may lead to an increase in 5d-5d overlap. Considering that Gd metal has somewhat higher $T_{\mathrm{c}}$ $(293.2 \mathrm{~K})$ [172], efforts in this direction are very promising. Wachter and Kaldis also confirmed this kind of effect of Gd-Gd distance [43]. Actually, [38] also reported that some other GdN derived alloys have $T_{\mathrm{c}}$ even higher than room temperature $(340 \mathrm{~K})$.

The other possibility for increasing $T_{\mathrm{c}}$ in the rare-earth pnictides is impurity doping. Magnetic impurities are helpful in assisting in the alignment of neighboring magnetic moments and they strengthen the magnetic interactions or even change the RKKY interaction to a long-range ferromagnetic interaction, thus dramatically enhancing $T_{\mathrm{c}}$. Examples can be found in [173], which reported that by adding a few per cent of magnetic ions such as $\mathrm{Mn}$, the $T_{\mathrm{c}}$ of $\mathrm{GaN}$ or $\mathrm{ZnO}$ well exceeds room temperature. Again mentioning the experiments on $\mathrm{GdN} / \mathrm{Fe}$ multilayers [127] in this context, it is worth noting that the Fe layers are found to be responsible for the long-range magnetic order in the GdN layer at temperatures largely above $T_{\mathrm{c}}$.

In addition, we noticed that some rare-earth pnictides of the $\mathrm{Th}_{3} \mathrm{P}_{4}$ type (or its anti-type) have very high $T_{\mathrm{c}}$ values, even higher than that of Gd metal. For example, the $T_{\mathrm{c}}$ of $\mathrm{Gd}_{4} \mathrm{Bi}_{3}$ is $340 \mathrm{~K}$ [174], for $\mathrm{Gd}_{4} \mathrm{Sb}_{3}$ it is $260 \mathrm{~K}$ [175], and for $\mathrm{Tb}_{4} \mathrm{Bi}_{3}$ it is $200 \mathrm{~K}$. The origin of these high $T_{\mathrm{c}} \mathrm{s}$ needs further investigation.

Despite their low transition temperatures, applications for the rare-earth pnictides do exist. For example, ErAs has been used to study magneto-transport [51] and resonant tunneling [176]; EuS has been used in hybrid spin filter devices [177, 178]. These materials are of special interest for using as barriers in magnetic tunnel junctions [179]. This is due to the exchange splitting of the conduction band which makes the barrier height spin dependent. Thus, given the exponential dependence of the tunneling current on barrier height, a highly spin-polarized current is expected. In a series of spin-dependent tunneling experiments, Moodera et al found a tunneling spin polarization of approximately $80 \%$ in $\mathrm{Al} / \mathrm{EuS} / \mathrm{M}$ junctions, where $\mathrm{M}$ was $\mathrm{Ag}, \mathrm{Au}$, or $\mathrm{Al}$ [180]. Using a related Eu chalcogenide, EuSe, they were able to demonstrate essentially $100 \%$ spin polarization [181]. New hybrid devices utilizing the spin filtering properties of rare-earth monopnictides are expected in the near future.

\section{Conclusions}

After more than 40 years' efforts, stimulated first by the search for magnetic semiconductors and now by spintronics materials, we now have a better understanding of the electronic, magnetic and transport properties of rare-earth monopnictides.

Rare-earth monopnictides are generally semiconductors or semimetals. Despite their simple rock salt structure, they demonstrate various types of magnetic ordering generally 
with low transition temperatures. Their electronic structures and magnetic properties are sensitive to temperature, pressure (strain) and impurity effects. The rare-earth $4 \mathrm{f}-5 \mathrm{~d}$ interactions and the hybridizations between the rare-earth non- $4 \mathrm{f}$ and pnictogen $\mathrm{p}$ states are responsible for many fascinating phenomena that occur in rare-earth monopnictides. In contrast to the rare-earth metals, both superexchange interaction and indirect RKKY-type interaction coexist in rare-earth monopnictides, and the former is dominant in heavy rareearth monopnictides. It is possible that other exchange coupling mechanisms, such as direct exchange and double exchange, may also be found in rare-earth monopnictides.

We conclude that the high-polarization, nominally half-metallic, rare-earth monopnictides are most likely to be found in rare-earth nitrides or nitrogen-involved rare-earth alloys. This is because those nitrides are on the border between semiconductor and metal due to their anion sizes. Impurity doping, structural distortion, and lattice constraint effects have a significant influence on the electronic, magnetic and transport properties of rareearth nitrides.

During the past several decades, various theoretical and experimental studies have helped to study the intriguing properties of these materials. Theoretically, much more powerful tools have been developed to solve the complicated yet fascinating many-body problems existing in these strongly correlated systems, such as SIC, LSDA + $U$, GWA and DMFT. With the fast increase in the computational power and the more mature massively parallel computing technology, there is more confidence we can have to deal with the necessary large scale calculations.

State-of-the-art crystal and film growth techniques make it possible now to obtain high quality samples to determine the electronic structure, the Fermi surfaces, and physical properties of these compounds. With improved samples, techniques like low energy electron-diffraction, scanning tunneling microscopy, angle-resolved (inverse) photoemission, neutron-diffraction are proving to be more helpful in characterizing the geometric, electronic and magnetic structures of both thin films and crystals, particularly when combined with element specific XMCD experiments.

Still, there are many unresolved issues related to the half-metallicity in rare-earth monopnictides and many blanks that need to be filled in table 1 . To address these issues or fill in the blanks, a close collaboration between experimentalists and theorists is absolutely necessary. Nevertheless, it takes a little imagination to conclude that we can expect further exciting results to emerge from the study of the electronic structure of rare-earth compounds.

\section{Acknowledgments}

The authors acknowledge insightful discussions with P. W. Anderson, D. S. Wang, A. G. Petukhov, W. Nolting, and A. Sharma. Special thanks are due to S. Granville, S. Suga, and D. X. Li, for giving permission to use the original figures from their publications. This work was supported by NSF (Grants DMR-0203359, MRSEC-DMR-0213808, and EPS-9720643), the Nebraska Research Initiative, and Department of the Army Grants DAAG 55-98-1-0273 and DAAG 55-991-0106 and the Office of Naval Research (Grant No. N00014-06-1-0616). The computational part of this work was completed utilizing the Research Computing Facility of the University of Nebraska-Lincoln.

\section{References}

[1] de Groot R A, Mueller F M, van Engen P G, and Buschow K H J 1983 Phys. Rev. Lett. 502024

[2] Wolf S A, Awschalom D D, Buhrman R A, Daughton J M, von Molnar S, Roukes M L, Chtchelkanova A Y, and Treger D M 2001 Science 2941488

[3] Pickett W E and Moodera J S 2001 Phys. Today 54 (May) 39 
[4] Dowben P A and Skomski R 2004 J. Appl. Phys. 957453

[5] Dowben P A and Jenkins S J 2005 Frontiers in Magnetic Materials ed A Narlikar (Berlin: Springer) p 295

[6] Chioncel L, Katsnelson M I, de Groot R A, and Lichtenstein A I 2003 Phys. Rev. B 68144425

[7] Irkhin V Y and Katsnelson M I 1985 J. Phys. C: Solid State Phys. 184173

[8] For a complete review, see the series: Gschneidner K A and Eyring L (ed) 1979 Handbook on Physics and Chemistry of Rare Earth (Amsterdam: North-Holland)

[9] Dowben P A, McIlroy D N, and Li D 1997 Handbook on the Physics and Chemistry of Rare Earths vol 24, ed K A Gschneidner and L Eyring (Amsterdam: North-Holland) chapter 159

[10] Cullity B D 1972 Introduction to Magnetic Materials (Reading, MA: Addison-Wesley) p 178

[11] Yakovkin I N, Komesu T, and Dowben P A 2002 Phys. Rev. B 66035406

[12] Olson C G, Chase S J, Canfield P, and Lynch D W 1998 J. Electron Spectrsoc. Relat. Phenom. 93175

[13] Andrews A B, Joyce J J, Arko A J, Thompson J D, Tang J, Lawrence J M, and Hemminger J C 1995 Phys. Rev. B $51 \mathrm{R} 3277$

[14] Guziewicz E, Durakiewicz T, Butterfield M T, Olson C G, Joyce J J, Arko A J, Sarrao J L, Moore D P, and Morales L 2004 Phys. Rev. B 69045102

[15] Guziewicz E, Durakiewicz T, Olson C G, Joyce J J, Butterfield M T, Arko A J, Sarrao J L, and Wojakowski A 2006 Surf. Sci. $\mathbf{6 0 0} 1632$

[16] Guziewicz E, Durakiewicz T, Oppeneer P M, Joyce J J, Thompson J D, Olson C G, Butterfield M T, Wojakowski A, Moore D P, and Arko A J 2006 Phys. Rev. B 73155119

[17] Anderson P W 1959 Phys. Rev. 1152

[18] Anderson P W 1978 Rev. Mod. Phys. 50191

[19] Van Vleck J H and Frank A 1929 Phys. Rev. 341494

[20] Van Vleck J H 1978 Rev. Mod. Phys. 50181

[21] Sclar N 1962 J. Appl. Phys. 33 2999; Sclar N 1964 J. Appl. Phys. 351534

[22] Child H R, Wilkinson M K, Cable J W, Koehler W C, and Wollan E O 1963 Phys. Rev. 131922

[23] Trammell G T 1963 Phys. Rev. 131932 and references therein

[24] Holtzberg F, McGuire T R, Methfessel S, and Suits J C 1964 Phys. Rev. Lett. 13 18; Holtzberg F, McGuire T R, Methfessel S, and Suits J C 1964 J. Appl. Phys. 35 1033; Holtzberg F, McGuire T R, Methfessel S, and Suits J C 1966 J. Appl. Phys. 37976

[25] Busch G 1967 J. Appl. Phys. 381386 and references therein

[26] Brown R F, Lawson A W, and Everett G E 1968 Phys. Rev. 172559

[27] McGuire T R, Gambino R J, Pickart S J, and Alperin H A 1969 J. Appl. Phys. 401009

[28] Birgeneau R J, Bucher E, Maita J P, Passell L, and Turberfield K C 1973 Phys. Rev. B 85345

[29] Narita A and Kasuya T 1985 J. Magn. Magn. Mater. 52373

[30] Takahashi H and Kasuya T 1985 J. Phys. C: Solid State Phys. 18 2697; Takahashi H and Kasuya T 1985 J. Phys. C: Solid State Phys. 182709

[31] Hasegawa A and Yanase A 1977 J. Phys. Soc. Japan 42492

[32] Slater J C 1951 Phys. Rev. 81385

[33] Narita A 1988 J. Phys. Soc. Japan 57 251; Narita A 1988 J. Phys. Soc. Japan 573961

[34] Xia J-B, Ren S-F, and Chang Y-C 1991 Phys. Rev. B 431692

[35] Petukhov A G, Lambrecht W R L, and Segall B 1994 Phys. Rev. B 507800

[36] Petukhov A G, Lambrecht W R L, and Segall B 1996 Phys. Rev. B 534324

[37] Kasuya T and Li D X 1997 J. Magn. Magn. Mater. 167 L1

[38] Rhyne J J and McGuire T R 1972 IEEE Trans. Magn. 8105

[39] Hulliger F 1979 Handbook on the Physics and Chemistry of Rare Earths vol 4, ed K A Gschneidner and L Eyring (Amsterdam: North-Holland) chapter 33, p 153

[40] Kaldis E 1974 J. Cryst. Growth 24/25 53

[41] G"untherodt G, Kaldis E, and Wachter P 1974 Solid State Commun. 151435

[42] Kaldis E and Zurcher C 1974 Helv. Phys. Acta 47421

[43] Wachter P and Kaldis E 1980 Solid State Commun. 34241

[44] Ruderman M A and Kittel C 1954 Phys. Rev. 9699

[45] Kasuya T 1956 Prog. Theor. Phys. (Kyoto) 1645

[46] Yosida K 1957 Phys. Rev. 106893

[47] Wachter P, Kaldis E, and Hauger R 1978 Phys. Rev. Lett. 401404

[48] Wachter P 1978 Phys. Rep. 44159

[49] Urban P, Jansen K, Sperlich G, and Davidov D 1978 J. Phys. F: Met. Phys. 8977 
[50] Palmstrøm C J, Tabatabaie N, and Allen S J 1988 Appl. Phys. Lett. 532608

[51] Allen S J, Tabatabaie N, Palmstrøm C J, Hull G W, Sands T, DeRosa F, Gilchrist H L, and Garrison K C 1989 Phys. Rev. Lett. 622309

[52] Degiorgi L, Bacsa W, and Wachter P 1990 Phys. Rev. B 42530

[53] Degiorgi L, Teraoka S, Compagnini G, and Wachter P 1993 Phys. Rev. B 475715

[54] Chattopadhyay T, Burlet P, Rossat-Mignod J, Bartholin H, Vettier C, and Vogt O 1994 Phys. Rev. B 4915096

[55] Waldfried C, Mcllroy D N, Li D, Pearson J, Bader S D, and Dowben P A 1995 Surf. Sci. 341 L1072

[56] Xiao J Q and Chien C L 1996 Phys. Rev. Lett. 761727

[57] Li D X, Haga Y, Shida H, Suzuki T, Koide T, and Kido G 1996 Phys. Rev. B 538473

[58] Li D X, Haga Y, Shida H, Suzuki T, and Kwon Y S 1996 Phys. Rev. B 5410483

[59] Yamada H, Fukawa T, Muro T, Tanaka Y, Imada S, Suga S, Li D-X, and Suzuki T 1996 J. Phys. Soc. Japan 65 1000

[60] Li D X, Haga Y, Shida H, Suzuki T, Kwon Y S, and Kido G 1997 J. Phys.: Condens. Matter 910777

[61] Jones R O and Gunnarsson O 1989 Rev. Mod. Phys. 61689

[62] Perdew J P and Zunger A 1981 Phys. Rev. B 235048 and references therein

[63] Cowan R D 1967 Phys. Rev. 16354

[64] Heaton R A, Harrison J G, and Lin C C 1983 Phys. Rev. B 285992

[65] Svane A and Gunnarsson O 1990 Phys. Rev. Lett. 651148

[66] Svane A and Gunnarsson O 1988 Phys. Rev. B 379919

[67] Strange P, Svane A, Temmerman W M, Szotek Z, and Winter H 1999 Nature 399756

[68] Petit L, Svane A, Szotek Z, and Temmerman W M 2003 Science 301498

[69] Svane A 1992 Phys. Rev. Lett. 681900

[70] Aerts C M, Strange P, Horne M, Temmerman W M, Szotek Z, and Svane A 2004 Phys. Rev. B 69045115

[71] Horne M, Strange P, Temmerman W M, Szotek Z, Svane A , and Winter H 2004 J. Phys.: Condens. Matter 16 5061

[72] Szotek Z, Temmerman W M, Svane A, Petit L, Strange P, Stocks G M, Ködderitzsch D, Hergert W, and Winter H 2004 J. Phys.: Condens. Matter 16 S5587

[73] Svane A, Kanchana V, Vaitheeswaran G, Santi G, Temmerman W M, Szotek Z, Strange P, and Petit L 2005 Phys. Rev. B 71045119

[74] Hubbard J 1963 Proc. R. Soc. A 276 238; Hubbard J 1964 Proc. R. Soc. A 277 237; Hubbard J 1964 Proc. R. Soc. A 281401

[75] Anisimov V I, Zaanen J, and Andersen O K 1991 Phys. Rev. B 44943

[76] Anisimov V I, Solovyev I V, Korotin M A, Czyzyk M T, and Sawatzky G A 1993 Phys. Rev. B 4816929

[77] Liechtenstein A I, Anisimov V I, and Zaanen J 1995 Phys. Rev. B 52 R5467

[78] Anisimov V I, Aryasetiawan F, and Lichtenstein A I 1997 J. Phys.: Condens. Matter 9767

[79] Anderson P W 1961 Phys. Rev. 12441

[80] Dudarev S L, Botton G A, Savrasov S Y, Humphreys C J, and Sutton A P 1998 Phys. Rev. B 571505

[81] Anisimov V I and Gunnarsson O 1991 Phys. Rev. B 437570

[82] Slater J C 1960 Quantum Theory of Atomic Structure vol 1 (New York: McGraw-Hill)

[83] Liechtenstein A I, Antropov V P, and Harmon B N 1994 Phys. Rev. B 4910770

[84] Komesu T, Jeong H-K, Choi J, Borca C N, Dowben P A, Petukhov A G, Schultz B D, and Palmström C J 2003 Phys. Rev. B 67035104

[85] Duan C-G, Komesu T, Jeong H-K, Choi J, Borca C N, Yin W-G, Liu J, Mei W N, Dowben P A, Petukhov A G, Schultz B D, and Palmstrøm C J 2004 Surf. Rev. Lett. 11531

[86] Blaha P, Schwarz K, Madsen G K H, Kvasnicka D, and Luitz J 2001 WIEN2k, An Augmented Plane Wave + Local Orbitals Program for Calculating Crystal Properties (Karlheinz Schwarz, Techn. Universität Wien, Austria) ISBN 3-9501031-1-2

[87] Duan C-G, Sabiryanov R F, Liu J, Mei W N, Dowben P A, and Hardy J R 2005 J. Appl. Phys. 97 10A915

[88] Duan C-G, Sabiryanov R F, Liu J, Mei W N, Dowben P A, and Hardy J R 2005 Phys. Rev. Lett. 94237201 Duan C-G, Sabiryanov R F, Liu J, Mei W N, Dowben P A, and Hardy J R 2006 Phys. Rev. Lett. 96139901

[89] Duan C-G, Sabiryanov R F, Mei W N, Jaswal S S, Dowben P A, and Tsymbal E Y 2006 Appl. Phys. Lett. 88 182505

[90] Anderson P W 1950 Phys. Rev. 79 350; Anderson P W 1959 Phys. Rev. 1152

[91] Johannes M D and Pickett W E 2005 Phys. Rev. B 72195116

[92] Ghosh D B, De M, and De S K 2005 Phys. Rev. B 72045140

[93] Larson P and Lambrecht W R L 2006 Phys. Rev. B 74085108 
[94] Kohn W and Sham L J 1965 Phys. Rev. 140 A1133

[95] Landau L D 1957 Sov. Phys._JETP 3 920; Landau L D 1957 Sov. Phys._JETP 5 101; Landau L D 1959 Sov. Phys.-JETP 3570

[96] Aryasetiawan F and Gunnarsson O 1998 Rep. Prog. Phys. 61237

[97] Hedin L 1965 Phys. Rev. 139 A796

[98] Pines D and Bohm D 1952 Phys. Rev. 85338

[99] Hybertsen M S and Louie S G 1985 Phys. Rev. Lett. 55 1418; Hybertsen M S and Louie S G 1988 Phys. Rev. B 372733

[100] Godby R W, Schlüter M and Sham L J 1986 Phys. Rev. Lett. 562415

[101] Onida G, Reining L, and Rubio A 2002 Rev. Mod. Phys. 74601

[102] van Schilfgaarde M, Kotani T, and Faleev S 2006 Phys. Rev. Lett. 96226402

[103] Lambrecht W R L 2000 Phys. Rev. B 6213538

[104] For a recent review, see Kotliar G, Savrasov S Y, Haule K, Oudovenko V S, Parcollet O, and Marianetti C A 2006 Rev. Mod. Phys. 78865

[105] Metzner W and Vollhardt D 1989 Phys. Rev. Lett. 62324

[106] Georges A, Kotliar G, Krauth W, and Rozenberg M J 1996 Rev. Mod. Phys. 6813

[107] Anisimov V I, Poteryaev A I, Korotin M A, Anokhin A O, and Kotliar G 1997 J. Phys.: Condens. Matter 9 7359

[108] Chitra R and Kotliar G 2000 Phys. Rev. B 6212715

[109] Lægsgaard J and Svane A 1998 Phys. Rev. B 5812817

[110] Sakai O, Shimizu Y, and Kaneta Y 2005 J. Phys. Soc. Japan 742517

[111] Kalvoda S, Dolg M, Flad H-J, Fulde P, and Stoll H 1998 Phys. Rev. B 572127

[112] Santos C, Nolting W, and Eyert V 2004 Phys. Rev. B 69214412

[113] Sharma A and Nolting W 2006 J. Phys.: Condens. Matter 187337

[114] Bhattacharjee S and Jaya S M 2006 Eur. Phys. J. B 49305

[115] Landrum G A, Dronskowski R, Niewa R, and DiSalvo F J 1999 Chem. Eur. J. 5515

[116] Marzari N and Vanderbilt D 1997 Phys. Rev. B 5612847

[117] Stengel M and Spaldin N A 2006 Phys. Rev. B 73075121

[118] Koyama K, Yoshida M, Nojiri H, Sakon T, Li D X, Suzuki T, and Motokawa M 2000 J. Phys. Soc. Japan 69 1521

[119] Koyama K, Yoshida M, Li D X, and Motokawa M 2001 J. Phys. Soc. Japan 702774

[120] Tomimatsu T, Koyama K, Yoshida M, Li D X, and Motokawa M 2003 Phys. Rev. B 67014406

[121] Nakanishi Y, Sakon T, Motokawa M, Ozawa M, and Suzuki T 2004 Phys. Rev. B 69024412

[122] Leuenberger F, Parge A, Felsch W, Fauth K, and Hessler M 2005 Phys. Rev. B 72014427

[123] Oliver M R, Kafalas J A, Dimmock J O, and Reed T B 1970 Phys. Rev. Lett. 241064

[124] Shapira Y, Foner S, Aggarwal R L, and Reed T B 1973 Phys. Rev. B 82316

[125] Leuenberger F, Parge A, Felsch W, Baudelet F, Giorgetti C, Dartyge E, and Wilhelm F 2006 Phys. Rev. B 73 214430

[126] Granville S, Ruck B J, Budde F, Koo A, Pringle D J, Kuchler F, Preston A R H, Housden D H, Lund N, Bittar A, Williams G V M, and Trodahl H J 2006 Phys. Rev. B 73235335

[127] Leuenberger F, Parge A, Felsch W, Neisius T, and Mathon O 2006 J. Appl. Phys. 100033905

[128] Olcese G L 1979 J. Phys. F: Met. Phys. 9569

[129] Smart J S 1966 Effective Field Theories of Magnetism (Philadelphia, PA: Saunders) pp 76-7

[130] Ono S, Nomura K, and Hayakawa H 1972 J. Less Common Met. 38119

[131] Iandelli A and Botti E 1976 Atti Accad. Naz. Lincei Cl. Sci. Fis. Mat. Nat. Rend. 254498

[132] Leger J M, Ravot D, and Rossat-Mignod J 1984 J. Phys. C: Solid State Phys. 174935

[133] Nomura K, Hayakawa H, and Ono S 1977 J. Less Common Met. 52259

[134] Schoenes J and Reim W 1985 J. Less Common Met. 11219

[135] Ettmayer P, Waldhart J, and Vendl A 1979 Mon. Chem. 1101109

[136] Jacobs H and Fink U 1978 Z. Anorg. Allg. Chem. 438151

[137] Mironov K E and Brygalina G P 1974 Izv. Akad. Nauk SSSR Neorg. Mater. 10787

[138] Bommeli F, Degiorgi L, and Wachter P 1995 J. Magn. Magn. Mater. 1401159

[139] Li D X, Oyamada A, Hashi K, Haga Y, Matsumura T, Shida H, Suzuki T, Kasuya T, Dönni A , and Hulliger F 1995 J. Magn. Magn. Mater. 1401169

[140] Klemm W and Winkelmann G 1956 Z. Anorg. Allg. Chem. 28887 
[141] Iandelli A 1964 Atti Accad. Naz. Lincei Cl. Sci. Fis. Mat. Nat. Rend. 37160

[142] Harmon B N, Antropov V P, Liechtenstein A I, Solovyev I V, and Anisimov V I 1995 J. Phys. Chem. Solids 561521

[143] Shick A B, Liechtenstein A I, and Pickett W E 1999 Phys. Rev. B 6010763

[144] Kurz Ph, Bihlmayer G, and Blügel S 2002 J. Phys.: Condens. Matter 146353

[145] Steglich F, Rauchschwalbe U, Gottwick U, Mayer H M, Sparn G, Grewe N, Poppe U, and Franse J J M 1985 J. Appl. Phys. $\mathbf{5 7} 3054$

[146] Rice T M and Ueda K 1985 Phys. Rev. Lett. 55995

[147] Monnier R, Rhyner J, Rice T M, and Koelling D D 1985 Phys. Rev. B 315554

[148] Ashcroft N W and Mermin N D 1976 Solid State Physics (New York: Holt, Rinehart, and Winston) p 652

[149] Galanakis I, Dederichs P H, and Papanikolaou N 2002 Phys. Rev. B 66174429

[150] Waldfried C, McAvoy T, Welipitiya D, Komesu T, Dowben P A, and Vescovo E 1998 Phys. Rev. B 587434

[151] Komesu T, Waldfried C, and Dowben P A 1999 Phys. Lett. A 25681

[152] Oleinik I I, Tsymbal E Y, and Pettifor D G 2002 Phys. Rev. B 65 R020401

[153] Velev J, Belashchenko K D, Stewart D, van Schilfgaarde M, Jaswal S S, and Tsymbal E Y 2005 Phys. Rev. Lett. 95216601

[154] Duan C-G, Jaswal S S, and Tsymbal E Y 2006 Phys. Rev. Lett. 97047201

[155] Dowben P A 2000 Surf. Sci. Rep. 40151

[156] Falicov L M and Kimball J C 1969 Phys. Rev. Lett. 22997

[157] Kreissl M and Nolting W 2005 Phys. Rev. B 72245117

[158] Harrison W A 1980 Electronic Structure and the Properties of Solids (San Francisco, CA: Freeman) pp 451, 481 and 505

[159] Singh D J 1994 Planewaves, Pseudopotentials and the LAPW Method (Boston, MA: Kluwer Academic) p 86

[160] Pittini R, Schoenes J, Vogt O, and Wachter P 1996 Phys. Rev. Lett. 77944

[161] Ghosh D B, De M, and De S K 2003 Phys. Rev. B 67035118

[162] Szade J and Drzyzga M 2000 J. Alloys Compounds 29972

[163] Drzyzga M, Szade J, Deniszczyk J, and Michalecki T 2003 J. Phys.: Condens. Matter 153701

[164] Weber F, Cosceev A, Nateprov A, Pfleiderer C, Faisst A, Uhlarz M, and Lohneysen H V 2005 Physica B 359 226

[165] Mozharivskyj Y and Franzen H F 2000 J. Solid State Chem. 152478

[166] Schneemeyer L F, van Dover R B, and Gyorgy E M 1987 J. Appl. Phys. 613543

[167] van Leuken H and de Groot R A 1995 Phys. Rev. Lett. 741171

[168] Pickett W E 1998 Phys. Rev. B 5710613

[169] Min B I, Park M S, and Park J H 2004 J. Phys.: Condens. Matter 16 S5509

[170] Akai H and Ogura M 2006 Phys. Rev. Lett. 97026401

[171] Kuznietz M 1971 J. Appl. Phys. 421470

[172] Nigh H E, Legvold S, and Spedding F H 1963 Phys. Rev. 1321092

[173] Coey J M, Venkatesan M, and Fitzgerald C B 2005 Nat. Mater. 4173

[174] Methfessel S and Kneller E 1963 Appl. Phys. Lett. 2115

[175] Holtzberg F, McGuire T R, Methfessel S, and Suits J C 1964 J. Appl. Phys. 351033

[176] Brehmer D E, Zhang K, Schwarz C J, Chau S P, and Allen S J 1995 Appl. Phys. Lett. 671268

[177] LeClair P, Ha J K, Swagten H J M, Kohlhepp J T, van de Vin C H, and de Jonge W J M 2002 Appl. Phys. Lett. 80625

[178] Filip A T, LeClair P, Smits C J P, Kohlhepp J T, Swagten H J M, Koopmans B, and de Jonge W J M 2002 Appl. Phys. Lett. 811815

[179] Tsymbal E Y, Mryasov O, and LeClair P R 2003 J. Phys.: Condens. Matter 15 R109

[180] Moodera J S, Hao X, Gibson G A, and Meservey R 1988 Phys. Rev. Lett. 61637

[181] Moodera J S, Meservey R, and Hao X 1993 Phys. Rev. Lett. 70853 